\title{
Water Pentamer: Characterization of the Torsional-Puckering Manifold by Terahertz VRT Spectroscopy
}

\author{
Heather A. Harker, ${ }^{\dagger}$ Mark R. Viant, $¥$ Frank N. Keutsch, ${ }^{\S}$ Ernest A. Michael, \\ Ryan P. McLaughlin, ${ }^{\perp}$ and Richard J. Saykally* \\ Department of Chemistry, University of California, Berkeley, California 94720
}

Received: March 23, 2005; In Final Form: April 29, 2005

\begin{abstract}
We present the measurement and analysis of five new $\left(\mathrm{D}_{2} \mathrm{O}\right)_{5}$ bands via vibration-rotation-tunneling (VRT) spectroscopy as well as a preliminary description of a second $\left(\mathrm{H}_{2} \mathrm{O}\right)_{5}$ band. The vibrationally averaged rotational constants of all five fitted bands agree well with those from the two previously observed $\left(\mathrm{D}_{2} \mathrm{O}\right)_{5}$ bands and confirm that the pentamer averages to a symmetric, quasi-planar structure on the time scale of our experiment. While the spectrum of the first two bands, located at $50.7 \mathrm{~cm}^{-1}(1.52 \mathrm{THz})$ and $27.3 \mathrm{~cm}^{-1}(0.82 \mathrm{THz})$ are indicative of unperturbed oblate rotors, the three remaining $\left(\mathrm{D}_{2} \mathrm{O}\right)_{5}$ bands centered at $47.7 \mathrm{~cm}^{-1}(1.43 \mathrm{THz})$, $45.4 \mathrm{~cm}^{-1}(1.36 \mathrm{THz})$, and $45.0 \mathrm{~cm}^{-1}(1.35 \mathrm{THz})$ are severely perturbed by first-order Coriolis coupling. This represents the first observation of this perturbation in the perdeuterated water pentamer, as well as the first observation of transitions between degenerate states of the torsional-puckering manifold. Unlike transitions from the $\left(\mathrm{H}_{2} \mathrm{O}\right)_{5}$ band observed by Brown et al. at $89.0 \mathrm{~cm}^{-1}$ and the $103.8 \mathrm{~cm}^{-1}$ band that we report here, none of the individual rovibrational transitions of any of the five $\left(\mathrm{D}_{2} \mathrm{O}\right)_{5}$ bands demonstrate spectral splittings due to bifurcation tunneling. We conclude, through careful analysis of these water pentamer bands, that at least three torsional-puckering manifolds have been probed and that the lowest-energy manifold is highly compacted. A plausible water pentamer torsional-puckering correlation diagram is proposed, though additional experimental data are required to unambiguously establish the energies of the torsional-puckering levels.
\end{abstract}

\section{Introduction}

Because it presents an alternative to the traditional tetrahedral structure as a means of achieving an ideal hydrogen bonding geometry, the water pentamer plays a special role in the hydrogen-bond network topologies of liquid water, larger water clusters, clathrate hydrates, and hydration shells of proteins and DNA. ${ }^{1,2}$ Ohmine showed that hydrogen-bond-network rearrangements (HBNR) in liquid water interconvert small aggregates such as the pentamer, hexamer, and so forth on a time scale much longer than that of the intermolecular vibrational modes of a typical water cluster and that identifiable five- and six-membered rings dominate the topology in the $\mathrm{HBN}{ }^{3}$ Collective motions of the HBN underlie the many unusual properties of liquid water, for example, the high melting and boiling points, the large heat capacity, and the density maximum at $4{ }^{\circ} \mathrm{C}$. Wales et al. have shown stacked pentameric rings to be prevalent in the global minima energy structures of larger water clusters, ${ }^{4}$ while $\mathrm{Ma}$ et al. demonstrated the presence of fused rings of puckered, cyclic water pentamers in crystalline hydrates,${ }^{5}$ with the pentamer structure being very similar to that determined by vibration-rotation-tunneling (VRT) spectroscopy, and Burnham et al. have found the global minimum

\footnotetext{
* To whom correspondence should be addressed: saykally@calmail.berkeley.edu.

† heatherharker@cal.berkeley.edu.

$\div$ Present address: School of Biosciences, The University of Birmingham, Birmingham, B15 2TT, U.K.; m.viant@bham.ac.uk.

$\S$ Present address: Department of Chemistry and Chemical Biology, Harvard University, Cambridge, MA 01238.; frank@huarp.harvard.edu.

"Present address: I. Physik Institut, Universität zu Köln, Germany.; michael@ph1.uni-koeln.de.

${ }^{\perp}$ Present address: Department of Chemistry, University of Washington, Seattle, WA 98195.; ryanmc@u.washington.edu.
}

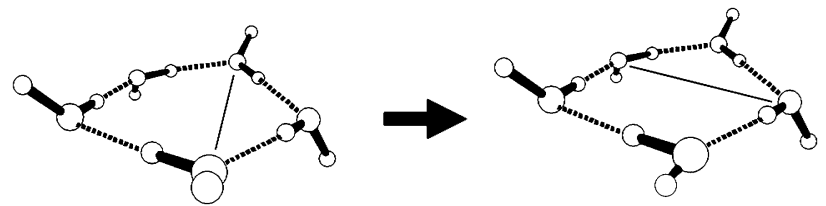

Figure 1. Torsional-puckering dynamics of the water pentamer. Flipping motion of a free hydrogen atom accompanied by the oxygen framework pucker pseudorotation; vibrational averaging of the $C_{1}$ equilibrium structure yields an effective $C_{5 h}$ structure.

pentamer ring structure to form readily even at liquid helium temperatures. ${ }^{6}$ Although infrared cavity ringdown spectroscopy of the gas-phase water pentamer reveals intramolecular absorption features of the gas-phase water pentamer that match the spectral features of the pentamer rings trapped inside helium droplets, as well as those of liquid water and amorphous ice, ${ }^{6-8}$ the IR absorptions appear as single narrow peaks presumably due to the floppy aspect of the puckered water pentamer oxygen framework that allows for enhanced coupling between the intraand intermolecular vibrational modes. In contrast, VRT spectroscopy directly probes the intermolecular vibrations and reveals the detailed fine structure that arises from quantum tunneling between degenerate minima.

The ab initio calculated equilibrium structure of the water pentamer is an asymmetric $\left(C_{1}\right)$, chiral homodromic ring, wherein each water molecule acts as both a single hydrogenbond donor and acceptor. The free hydrogen atom positions alternate above and below the oxygen ring framework (Figure 1). ${ }^{9}$ While the pentamer structure may appear to be a direct extension of that of the trimer, theory indicates that the pentamer 

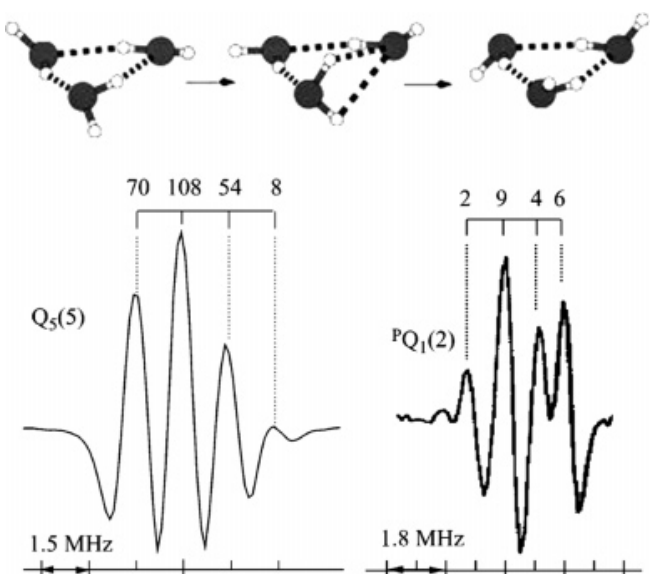

Figure 2. Bifurcation tunneling splittings in the water trimer- $d_{6}$. Exchange of a free and a bound hydrogen atom on an individual monomer is accompanied by the flipping of the free hydrogen atoms on the neighboring water molecules. This is the lowest-energy hydrogen-bond breaking motion, observed in the dimer, hexamer, and all cyclic water clusters except the water tetramer, and results in each rovibrational transition of the water trimer splitting into a quartet, with relative intensities determined by nuclear spin statistics. ${ }^{20,21}$

oxygen framework is puckered by $13^{\circ}$ or more, ${ }^{10}$ whereas the trimer framework is obviously planar. Terahertz VRT experiments on the water pentamer have shown that vibrational averaging via the facile torsional flipping of free hydrogen atoms is accompanied by pseudorotation of the ring pucker, resulting in a planar, symmetric $\left(C_{5 h}\right)$ effective structure (Figure 1). ${ }^{2,11,12}$ We note here that the water pentamer torsional-puckering energy manifold may elsewhere have been referred to simply as a torsional or pseudorotational manifold, but that the designation of "torsional-puckering" manifold better distinguishes this manifold from that of the water trimer.

Liu et al. observed the first $\left(\mathrm{D}_{2} \mathrm{O}\right)_{5}$ VRT band in 1997 by Terahertz laser spectroscopy. ${ }^{13}$ Whereas previous high-resolution VRT experiments on the water dimer, ${ }^{14}$ trimer, ${ }^{15}$ and hexamer ${ }^{16}$ had shown that HBNR dynamics occurring within these water clusters were manifested as small tunneling splittings of each rovibrational transition (Figure 2), the parallel $(\Delta K=0)$ band, measured by Liu et al. at $81.2 \mathrm{~cm}^{-1}$, as well as an additional parallel band measured shortly thereafter at $30.2 \mathrm{~cm}^{-1}$ by Cruzan et al., ${ }^{11}$ were both devoid of observable bifurcation tunneling splittings. It was not until Brown et al. reported the first $\left(\mathrm{H}_{2} \mathrm{O}\right)_{5}$ band at $89.0 \mathrm{~cm}^{-1}$, wherein each rovibrational transition was split into four observable lines separated by $4.8 \mathrm{MHz}$, that the bifurcation tunneling splittings, predicted by theoretical efforts, ${ }^{17-19}$ were observed in the water pentamer (Figure 3). ${ }^{12}$

The observed bifurcation tunneling splittings in the $\left(\mathrm{H}_{2} \mathrm{O}\right)_{5}$ water pentamer were, however, 60 times smaller than those of the typical torsional $\left(\mathrm{H}_{2} \mathrm{O}\right)_{3}$ trimer (Table 1). This is not surprising given the greater hydrogen-bond strength in the water pentamer and the coupling of the free hydrogen flipping motion, as well as bifurcation tunneling to the ring pucker pseudorotation, which requires accompanying motion of the heavy oxygen atom framework (Figure 1). Given that the bifurcation splitting decreases by a factor of $8-300$ going from the H6 to the D6 water trimer and that the analogous splitting in the $\mathrm{H} 10$ water pentamer is at best 5 times the spectral resolution of the terahertz spectrometer, it seems improbable that bifurcation tunneling splittings will be observed for the perdeuterated water pentamer. While this generates a simpler spectrum, it obviates both a powerful cluster identification tool and valuable dynamical information.
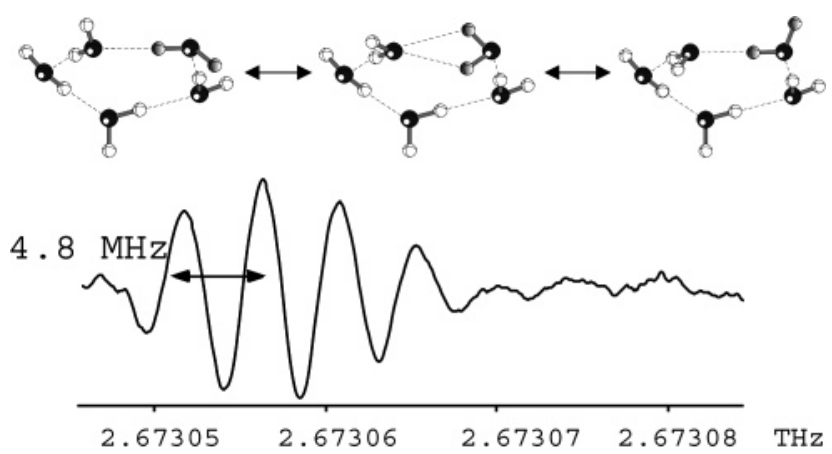

Figure 3. Bifurcation tunneling splittings in the $89.0 \mathrm{~cm}^{-1} \mathrm{H}_{2} \mathrm{O}$ water pentamer band. The exchange of a free and a bound hydrogen atom is shown accompanied by the flipping of the free hydrogen atom on the neighboring water molecule whose oxygen atom is the hydrogen-bond acceptor of the bifurcated transition state. In contrast to the water trimer, flipping of free hydrogen atoms, as well as bifurcation tunneling in the pentamer, requires a simultaneous pseudorotation of the oxygen framework pucker (Figure 1). Note: Only four tunneling components were observed because of nuclear spin statistics and degeneracies.

TABLE 1: Observed Bifurcation Tunneling Splittings and Predicted Barriers of Torsional Bands

\begin{tabular}{|c|c|c|c|c|}
\hline & $\left(\mathrm{H}_{2} \mathrm{O}\right)_{3}$ & $\left(\mathrm{D}_{2} \mathrm{O}\right)_{3}$ & $\left(\mathrm{H}_{2} \mathrm{O}\right)_{5}$ & $\left(\mathrm{D}_{2} \mathrm{O}\right)_{5}$ \\
\hline barrier $\left(\mathrm{cm}^{-1}\right)$ & \multicolumn{2}{|c|}{$819^{a}$} & \multicolumn{2}{|c|}{$1112^{b}$} \\
\hline splitting (MHz) & $40-300^{c}$ & $<1-5^{c}$ & $5^{d}$ & not obsd \\
\hline
\end{tabular}

${ }^{a}$ ref $21 .{ }^{b}$ ref 22. ${ }^{c}$ ref $23 .{ }^{d}$ ref 12.

The $89.0 \mathrm{~cm}^{-1}\left(\mathrm{H}_{2} \mathrm{O}\right)_{5}$ band provided not only the first analysis of water pentamer bifurcation tunneling dynamics but also the first insight into its Coriolis perturbations. Coriolis coupling had already been observed in the dimer, ${ }^{14}$ trimer, ${ }^{23}$ and tetramer, ${ }^{24}$ but first-order Coriolis splittings, such as those in the $\left(\mathrm{H}_{2} \mathrm{O}\right)_{5}$ pentamer band, were originally predicted to be vanishingly small ${ }^{25,26}$ for cyclic water clusters. Nonetheless, analysis of the extensive water trimer data set, with its linear Coriolis splittings, had already forced a revision of this thinking. Indeed, both first- and second-order Coriolis effects in the water trimer were predicted by the torsional model of van der Avoird et al. if the effective Hamiltonian accounted for Coriolis coupling through second order. ${ }^{27,28}$ First-order perturbation theory predicts no first-order Coriolis effects in the water trimer, because the pseudorotation of the cluster does not produce any internal (vibrational) angular momentum. Once Viant et al. employed both the unexpected first-order and the second-order Coriolis effects to characterize the perturbed water trimer bands, a highly accurate (root-mean-sqare $(\mathrm{rms})=1.36 \mathrm{MHz})$ global fit of 9 $\left(\mathrm{D}_{2} \mathrm{O}\right)_{3}$ pseudorotational bands comprising 554 individual VRT transitions was obtained (Figure 4). ${ }^{29}$ Brown et al. quickly followed with a global fit of the $\left(\mathrm{H}_{2} \mathrm{O}\right)_{3}$ data, ${ }^{30}$ then subsequently used first-order Coriolis effects to successfully fit the $89.0 \mathrm{~cm}^{-1}$ $\left(\mathrm{H}_{2} \mathrm{O}\right)_{5}$ band, noting however that second-order Coriolis terms were not needed. ${ }^{12}$

We report here the analysis of five new $\left(\mathrm{D}_{2} \mathrm{O}\right)_{5}$ bands, three of which exhibit a linear Coriolis splitting similar to that of the $\left(\mathrm{H}_{2} \mathrm{O}\right)_{5}$ band. This is the first observation of such splittings in the fully deuterated water pentamer and represents an important step toward quantifying the torsional-puckering eigenstate manifold, which is the direct consequence of quantum tunneling between degenerate minima by a hindered rotation. Indeed, in the case of the water trimer, the simple symmetric rotor Hamiltonian revealed that, for transitions involving only nondegenerate energy levels, no Coriolis perturbations are expected, given that they arise from second-order terms. We therefore propose that these transitions are also the first observed 

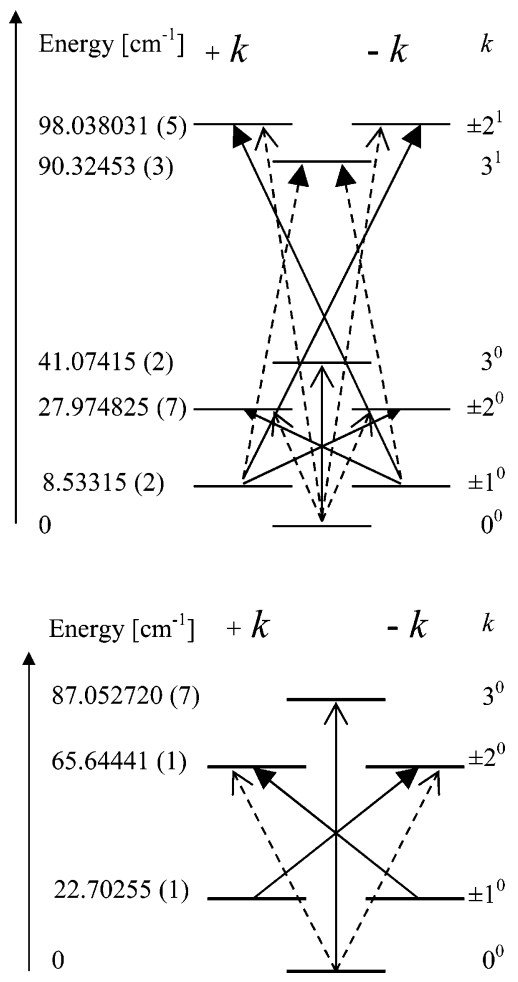

Figure 4. Experimentally determined torsional-puckering energy manifolds of the water trimer. Top: $\left(\mathrm{D}_{2} \mathrm{O}\right)_{3}$; includes 1 bands comprising 659 individual transitions..$^{23} k$ is the pseudorotational quantum number. Dashed arrows correspond to perpendicular bands $(\Delta K= \pm 1)$, solid arrows to parallel bands $(\Delta K=0)$. Open arrowheads correspond to transitions from the vibrational ground state, closed arrows to hot-band transitions from the $k= \pm 1^{0}$ level. Bottom: $\left(\mathrm{H}_{2} \mathrm{O}\right)_{3}$; includes 5 bands comprising 361 transitions. ${ }^{23}$ All bands involving degenerate $k= \pm n$ levels are severely perturbed by Coriolis coupling of the torsional flipping motion of the free $\mathrm{H}$ (D) atoms with the overall rotation of the cluster.

transitions of the $\mathrm{D}_{2} \mathrm{O}$ pentamer that connect degenerate energy levels within the torsional-puckering manifold and that they are necessarily "hot bands," in the sense that they do not arise from the vibrational ground state.

\section{Theoretical Considerations}

A thorough treatment of the perdeuterated water pentamer quantum tunneling dynamics has been given elsewhere..$^{2,11,13}$ Here, we provide only a brief review of the material essential for an understanding of the spectra and dynamics of the torsional-puckering energy level manifold. In 1996, Wales and Walsh (WW) produced a detailed group theoretical treatment of the water pentamer that identified the two lowest-energy rearrangement pathways: ${ }^{18}$ flipping or torsion of the free hydrogen atoms (Figure 1) and bifurcation or donor tunneling (Figure 3). The hydrogen flipping motion in the pentamer links degenerate minima via quantum tunneling and is executed by one of the two neighboring monomers located on the same side of the oxygen ring framework. The free hydrogen undergoes an odd number of flips, and since the pentamer ring is puckered, the oxygen framework must simultaneously rearrange in order to reach a degenerate minimum energy structure. The pucker phase can be shown to move around the oxygen ring in the same direction as the flipping motion. Each consecutive hydrogen flip rotates the principal inertial axis system by $-2 \pi / 5$ rad around the $z$ axis, which is directed out of the quasi-planar oxygen framework and toward the side of the ring with two free hydrogen atoms. Thus, the motion created by sequential

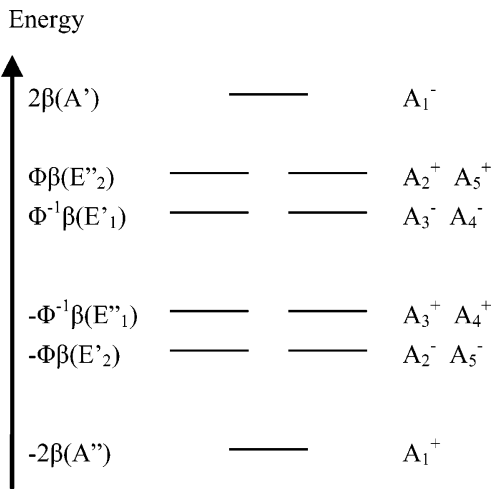

Figure 5. Torsional-puckering energy manifold of the water pentamer. Relative splittings are symmetrically equivalent to the Hückel model of 10 -annulene (cyclodecapentaene). $\beta$ is the appropriate tunneling matrix element, $\Phi=(\sqrt{ } 5+1) / 2$ is the golden ratio, and $\Phi^{-1}=(\sqrt{5}$ - 1)/2 is its reciprocal. Symmetry species, in parentheses, are appropriate if the generator corresponds to the operation $S_{5}$ of the molecular symmetry (MS) group $C_{5 h}$.

hydrogen flips is symmetrically equivalent to the external rotation of the cluster about the $z$ axis and is often referred to as a "pseudorotation".

As for the case of the trimer, the vibrationally averaged symmetric rotor behavior observed in the THz-VRT data for all the upper and lower states and ab initio results ${ }^{10,18,31,32}$ suggests that this pseudorotation must be responsible for the observed rovibrational bands, leading to a pseudorotational manifold similar to that of the trimer. However, whereas six sequential free hydrogen flips are required to sample the six degenerate minimum energy structures of the trimer, ten consecutive free hydrogen torsions accompanied by a ring puckering motion are necessary in the case of the pentamer. The first ten torsional-puckering energy levels generated by this pseudorotation, as calculated by WW using a Hückel-type approximation, ${ }^{18}$ are shown in Figure 5. Eight of the ten calculated energy levels are rigorously degenerate in this model, since Coriolis effects were not included. $\beta$ is empirically calculable within this model for both the water trimer and the water pentamer as one-fourth of the negative transition frequency $k=3^{0} \leftarrow 0^{0}$ (viz. one-fourth of -41.1 and $-87.1 \mathrm{~cm}^{-1}$ for $\left(\mathrm{D}_{2} \mathrm{O}\right)_{3}$ and $\left(\mathrm{H}_{2} \mathrm{O}\right)_{3}$, respectively) and one-fourth of the negative transition frequency for $k=5^{0} \leftarrow 0^{0}$ for the water pentamer. (Note: Levels with a 0 superscript were originally referred to as "lower," and levels with a 1 superscript as "upper.")

The model of WW provided a reasonable first approximation to the torsional-puckering energy manifold; however, Liu et al. ${ }^{13}$ argued that the perturbative tunneling model of WW was insufficiently developed to fully characterize the hindered rotational motions of the water pentamer. Liu et al. indicated two major shortcomings of the tunneling model of WW: (1) It only predicted a number of energy levels equal to the order of the MS group, nominally ten, since splittings beyond the ground state were not included. A quick glance at the known torsional energy manifold of the $\mathrm{D}_{2} \mathrm{O}$ water trimer (Figure 4) illustrates the problem with this restriction. The model of WW thereby only predicted transition frequencies lower in energy than the maximum tunneling splitting of the torsional-puckering manifold, $4|\beta|$, and predicted only one manifold. As will be explained in detail later, the existence of three distinct torsional bands arising from transitions that originate and terminate in degenerate states proves the necessity for multiple torsional-puckering energy manifolds to describe the observed transitions of the water pentamer. (2) The rotational selection rules of the 
WW model did not distinguish between the $\Delta K=+1$ and $\Delta K$ $=-1$ transitions, where $K$ is the projection of the rotational quantum number $J$ along the $z$ axis, that one important in assigning perpendicular bands involving Coriolis perturbations such as those found in the trimer. Brown et al. have already described one Coriolis perturbed $\left(\mathrm{H}_{2} \mathrm{O}\right)_{5}$ band, ${ }^{12}$ and here, we present three more bands for the perdeuterated water pentamer.

Liu et al. proposed ${ }^{13}$ an extension of the trimer model developed by van der Avoird et al. ${ }^{27}$ to better account for the flipping-puckering dynamics of the water pentamer, as well as the Coriolis coupling of these dynamics to the overall rotation of the cluster. The resulting overall pseudorotational selection rule is simply $\Delta K-\Delta k=5$ (modulo 10), yet the detailed rotational selection rules for perpendicular bands, $\Delta K= \pm 1$, now differ. For example, the selection rules for $\Delta k=-4\left(\mathrm{~A}_{2}{ }^{+}\right)$ $\leftarrow 0\left(\mathrm{~A}_{1}^{+}\right)$and $\Delta k=+4\left(\mathrm{~A}_{5}^{+}\right) \leftarrow 0\left(\mathrm{~A}_{1}^{+}\right)$are distinguishable regardless of the accidental degeneracy of the $\Delta k= \pm 4$ levels. This practical distinction becomes increasingly important to spectroscopists, as Coriolis coupling lifts the accidental degeneracy of the $\Delta k= \pm n$ levels.

Liu et al. then used a 5-D pseudorotational Hamiltonian similar to that formulated for the trimer to calculate the ten lowest pseudorotational states of the fully deuterated pentamer. The five degrees of freedom correspond to the five internal rotational angles of the flipping monomers, the coupling between pseudorotation and overall cluster rotation is neglected, and perhaps most importantly, the effect of puckering on the reduced internal rotational constant is ignored. In fact, Liu et al. deduced the energies of the lower pentamer manifold by direct scaling of the relative trimer state energies, arguing that, while the lower pentamer manifold might be compressed in comparison to that of the trimer due to heavy oxygen ring puckering motions, this effect would be counterbalanced by the reduced ring strain and additional vibrational degrees of freedom afforded the pentamer. The separation, $\Delta E$, between the lowest torsional manifold, $5^{0}$, and the next highest torsional manifold, $5^{1}$, was then crudely estimated, again through direct analogy with the trimer (Figure 6). Although the exact value of this separation, $\Delta E=49.3 \mathrm{~cm}^{-1}$, for the $\mathrm{D}_{2} \mathrm{O}$ trimer was not known at the time, Olthof et al. had correctly estimated it to be slightly larger than the known $k=$ $3^{0} \leftarrow 0^{0}$ splitting of $41.1 \mathrm{~cm}^{-1} .{ }^{28}$ The resulting torsional energy manifold of $\left(\mathrm{D}_{2} \mathrm{O}\right)_{5}$ is shown schematically in Figure 6 alongside the experimentally determined torsional energy manifold of $\left(\mathrm{D}_{2} \mathrm{O}\right)_{3}$. In light of this comparison, it seems quite reasonable for Liu et al. to have assigned the $81.2 \mathrm{~cm}^{-1}$ band to the $k=5^{1}$ $\leftarrow 0^{0}$ transition, especially since the analogous treatment of $\left(\mathrm{H}_{2} \mathrm{O}\right)_{5}$ found the $89.0 \mathrm{~cm}^{-1}$ band of Brown et al. to coincide with the predicted $k=5^{0} \leftarrow 0^{0}$ parallel transition. This was, however, the extent of the experimental data available to Liu et al. Cruzan et al. subsequently published the results for the parallel $30.2 \mathrm{~cm}^{-1}\left(\mathrm{D}_{2} \mathrm{O}\right)_{5}$ band ${ }^{11}$ but avoided discussion of its assignment within the torsional-puckering energy manifold. Cruzan subsequently proposed ${ }^{33}$ that this band could either be assigned to the $k=5^{0} \leftarrow 0^{0}$ transition, in accord with the model of Liu et al., or both the 30.2 and $81.2 \mathrm{~cm}^{-1}$ bands could be shifted to still higher energy in the manifolds; this would result in a very compact lower manifold of states, in better accord with the predictions of WW that placed $k=5^{0}$ at only 12.8 $\mathrm{cm}^{-1}$ above the ground state $k=0^{0}$.

Before any further experimental data became available, Graf et al. published an ab initio study on the torsional-puckering pseudorotation in the cyclic water pentamer ${ }^{31}$ that called into question the model of Liu et al. and added credibility to the model of a compact lower manifold of states. Graf et al. began

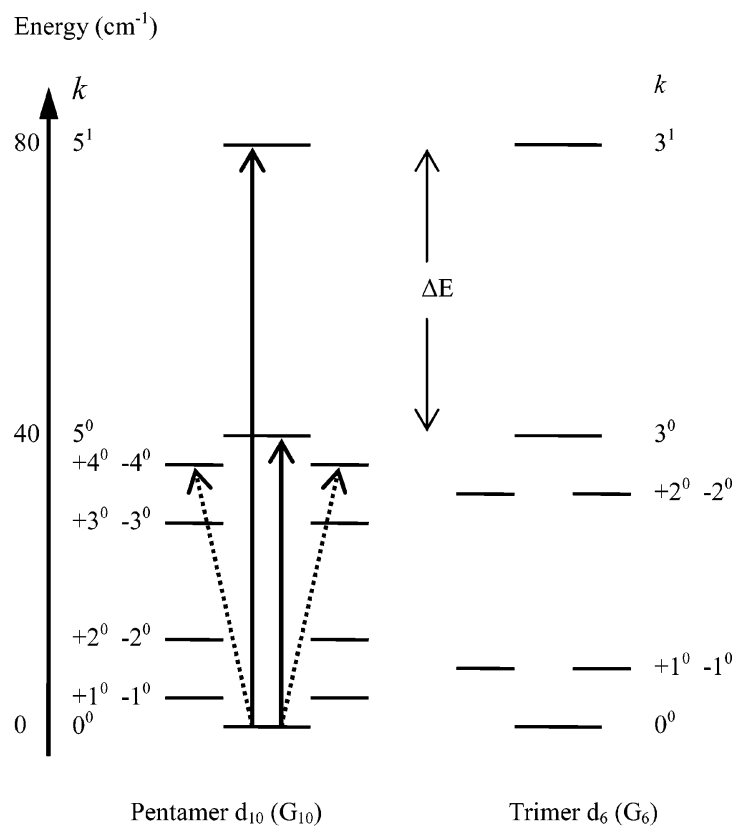

Figure 6. Estimated $\left(\mathrm{D}_{2} \mathrm{O}\right)_{5}$ torsional-puckering energy manifold compared to the confirmed $\left(\mathrm{D}_{2} \mathrm{O}\right)_{3}$ torsional manifold. Two types of strongly allowed transitions are superimposed on the water pentamer manifold: perpendicular transitions, $\Delta K= \pm 1$ (dashed) and parallel transitions $\Delta K=0$ (solid). Coriolis coupling is expected to split the accidentally degenerate $\Delta k= \pm n$ levels.

by investigating the intermolecular potential energy surface of the cyclic homodromic water pentamer at the MP2/aug-ccpVDZ level. Three stationary points were identified: (i) the puckered global minimum structure (GS), (ii) a torsionalpuckering saddle point (TS1), $62 \mathrm{~cm}^{-1}$ higher, and (iii) a planar $C_{5 h}$ symmetric structure $800 \mathrm{~cm}^{-1}$ higher in energy. The lowest normal-mode frequencies and intensities calculated for the GS structure of $\left(\mathrm{D}_{2} \mathrm{O}\right)_{5}$ are listed in Table 2 .

Through careful comparison of the lowest-frequency modes at GS and TS1, Graf et al. concluded that the torsional-puckering motion could not be described either as a pure $\mathrm{O}-\mathrm{H}$ torsional motion, as in the water trimer and Liu's analogous treatment of the pentamer, or as a pure pseudorotation of the pucker (as in cyclopentane), but rather comprised both types of coordinates. Furthermore, Graf et al. noted that the fractional contributions of the torsional and puckering motions changed along the torsional-puckering minimum energy path. Near GS, the first four modes can be described as predominantly out-of-plane and in-plane ring deformations of almost purely translational character, the next three modes as quasi-pure torsional motions, and the eighth mode as an intermolecular ring-breathing motion. It should be noted that the frequency of this ring-stretching motion is lower for $\left(\mathrm{H}_{2} \mathrm{O}\right)_{5}$, located between the ring deformations and the torsional motions. Experimentally, the hydrogenbond stretch motions of the fully deuterated water dimer and trimer have been located near $140 \mathrm{~cm}^{-1}, 34,35$ with larger clusters presumably exhibiting these transitions at somewhat higher frequencies.

Graf et al. then used the potential energies of GS and TS1 to define a one-dimensional cyclic torsional-puckering potential with tenfold symmetry. They found that the total pseudorotational path length, while longer for $\left(\mathrm{D}_{2} \mathrm{O}\right)_{5}$ than for $\left(\mathrm{H}_{2} \mathrm{O}\right)_{5}$, increased by only a factor of 1.30 compared to the factor of 2 that would be expected if the motion were purely torsional, as for the trimer. In fact, this factor of 1.3 is much closer to the factor of $\sqrt{ } 2$ that would be expected in the case of a vibration. They also found that their reduced internal rotational constants, 
TABLE 2: Harmonic Vibrational Normal Modes of the Ground-State Structure of $\left(\mathrm{D}_{2} \mathrm{O}\right)_{5}$, Calculated at the MP2/aug-cc-pVDZ Level $^{a}$

\begin{tabular}{|c|c|c|c|c|c|c|c|c|}
\hline \multirow[b]{2}{*}{ mode } & \multirow{2}{*}{$\begin{array}{l}\text { frequency } \\
\left(\mathrm{cm}^{-1}\right)\end{array}$} & \multirow{2}{*}{$\begin{array}{l}\text { intensity } \\
(\mathrm{km} / \mathrm{mole})\end{array}$} & \multirow[b]{2}{*}{ description } & \multicolumn{2}{|c|}{ translation } & \multicolumn{2}{|c|}{ rotation } & \multirow[b]{2}{*}{ intramolecular } \\
\hline & & & & $x y$ & $z$ & $z$ & $x y$ & \\
\hline 1 & 21.6 & 3.08 & oop deformation/torsion & 2.5 & 82.7 & 0.1 & 14.7 & 0 \\
\hline 2 & 39.7 & 0.006 & oop deformation/torsion & 4.7 & 84.7 & 0 & 10.5 & 0 \\
\hline 3 & 60.8 & 0.454 & ip deformation & 95 & 0.5 & 2.2 & 2.3 & 0 \\
\hline 4 & 63.1 & 0.709 & ip deformation & 96.3 & 0.9 & 2.4 & 0.4 & 0 \\
\hline 5 & 141.4 & 23.2 & torsion & 1.1 & 1.5 & 1.4 & 95.8 & 0.2 \\
\hline 6 & 145.8 & 20.1 & torsion & 3.2 & 2.6 & 2.2 & 91.7 & 0.3 \\
\hline 7 & 166.4 & 53.7 & torsion & 28.6 & 1.6 & 1.4 & 68.3 & 0.1 \\
\hline 8 & 174.6 & 29.1 & ip "symmetric" stretch & 67.7 & 3.4 & 0.6 & 28.2 & 0.1 \\
\hline 9 & 193.8 & 62.3 & torsion & 14.4 & 3.8 & 1.4 & 80.3 & 0.1 \\
\hline 10 & 219.2 & 11.8 & torsion & 33.6 & 0.9 & 2.1 & 63.3 & 0.1 \\
\hline 11 & 228.8 & 103 & ip stretch/torsion & 78.3 & 2.6 & 6.7 & 12.3 & 0.1 \\
\hline 12 & 237.4 & 115 & ip stretch/torsion & 54.4 & 2.9 & 4.7 & 37.8 & 0.1 \\
\hline
\end{tabular}

${ }^{a}$ The energy partitioning into translational, rotational, and monomer deformation is also given.

TABLE 3: Calculated Eigenvalues $\left(\mathrm{cm}^{-1}\right)$ for Cyclic $\left(\mathrm{H}_{2} \mathrm{O}\right)_{5}$ and $\left(\mathrm{D}_{2} \mathrm{O}\right)_{5}$ from the Effective One-Dimensional Torsional-Puckering Pseudorotational Model

\begin{tabular}{cccccc}
\hline$k$ & $\left(\mathrm{H}_{2} \mathrm{O}\right)_{5}$ & $\left(\mathrm{D}_{2} \mathrm{O}\right)_{5}$ & $k$ & $\left(\mathrm{H}_{2} \mathrm{O}\right)_{5}$ & $\left(\mathrm{D}_{2} \mathrm{O}\right)_{5}$ \\
\hline $\mathrm{ZPE}$ & -25.43 & -22.577 & $0^{1}$ & 138.41 & 84.87 \\
$0^{0}$ & 0 & 0 & $0^{2}$ & 153.47 & 102.61 \\
$\pm 1^{0}$ & 1.18 & 0.56 & $\pm 1^{2}$ & 175.71 & 112.86 \\
$\pm 2^{0}$ & 4.64 & 2.14 & $\pm 2^{2}$ & 206.84 & 130.39 \\
$\pm 3^{0}$ & 9.98 & 4.41 & $\pm 3^{2}$ & 241.15 & 150.36 \\
$\pm 4^{0}$ & 15.97 & 6.64 & $\pm 4^{2}$ & 278.36 & 172.23 \\
$5^{0}$ & 19.09 & 7.63 & $5^{2}$ & 317.61 & 194.51 \\
$5^{1}$ & 58.09 & 45.37 & $5^{3}$ & 319.24 & 197.39 \\
$\pm 4^{1}$ & 63.94 & 47.93 & $\pm 4^{3}$ & 361.31 & 221.4 \\
$\pm 3^{1}$ & 78.1 & 54.85 & $\pm 3^{3}$ & 406.98 & 248.48 \\
$\pm 2^{1}$ & 97 & 64.8 & $\pm 2^{3}$ & 455.44 & 277.26 \\
$\pm 1^{1}$ & 119.14 & 76.52 & & &
\end{tabular}

which included the puckering motion in addition to the torsional motion, were over 1 order of magnitude smaller than those used by Liu et al., which referred to the torsional motion only. The eigenvalues of the 1-D Schrödinger equation based on this potential are shown in Table 3. (Note: Graf et al. originally employed yet another pseudorotational quantum numbering system wherein the lowest torsional-puckering energy manifold was labeled $k=0-5$, the second lowest $k=5-10$, etc.) As expected for the MS group $\mathrm{G}_{10},{ }^{18}$ the torsional-puckering energy levels occur in the following sequence: a nondegenerate ground state, followed by four doubly degenerate states, then another nondegenerate state, transforming as $\mathrm{A}^{\prime}, \mathrm{E}_{2}{ }_{2}, \mathrm{E}_{1}^{\prime}, \mathrm{E}_{1}{ }_{1}, \mathrm{E}_{2}{ }_{2}$, and $\mathrm{A}^{\prime \prime}$, respectively ( $C_{5 h}$ nomenclature) (refer to Figure 5 ).

However, in contrast to the model of Liu et al., a compact lowest manifold is predicted for both $\left(\mathrm{D}_{2} \mathrm{O}\right)_{5}$ and $\left(\mathrm{H}_{2} \mathrm{O}\right)_{5}$, in accord with the earlier predictions of WW that placed $k=5^{0}$ at 12.8 and $18.0 \mathrm{~cm}^{-1}$ above the ground state, $k=0^{0}$, for $\left(\mathrm{D}_{2} \mathrm{O}\right)_{5}$ and $\left(\mathrm{H}_{2} \mathrm{O}\right)_{5}$, respectively. It is noteworthy that the calculated MP2/aug-ccpVDZ counterpoise-uncorrected barrier height of $81.2 \mathrm{~cm}^{-1}$ for $\left(\mathrm{D}_{2} \mathrm{O}\right)_{5}$ leads to a high-barrier situation, which Liu et al. argued against. Furthermore, Gregory and Clary studied the reaction path of the water pentamer calculated by Wales and Walsh ${ }^{17,18}$ and concluded that the torsional-puckering tunneling splitting would decrease by 2 orders of magnitude when going from the water trimer to the water pentamer because of the movement of oxygen atoms and consequently longer mass-weighted path length. ${ }^{32}$ Clearly, Liu et al. may have underestimated the influence of the oxygen ring puckering on water pentamer dynamics.

Graf et al. presented a graphical depiction of the calculated torsional-puckering energy levels as a function of barrier height, wherein the effective barrier height was varied from 0 to 250 $\mathrm{cm}^{-1}$, about three times the predicted barrier height. This is

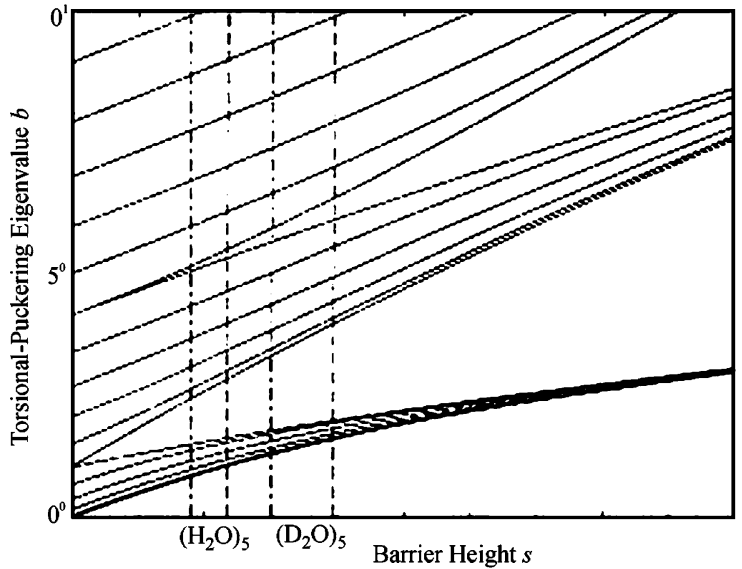

Figure 7. Dependence of torsional-puckering eigenstates of the cyclic water pentamer on barrier height. Dimensionless; shows the reduced pseudorotation state energies $(b)$ vs the reduced barrier height $(s){ }^{31}$ The dot - dashed and dashed vertical lines indicate the respective $\mathrm{CP}$ corrected and noncorrected barrier heights predicted for $\left(\mathrm{H}_{2} \mathrm{O}\right)_{5}$ and $\left(\mathrm{D}_{2} \mathrm{O}\right)_{5}$

reproduced below in Figure 7. Not only does the lower torsionalpuckering energy manifold become increasingly compressed as the barrier height to the torsion-puckering motion increases, but so do the other torsional manifolds, albeit to a lesser degree. This leads to a compact lower manifold, as well as to an increased separation between the various manifolds.

There are immediate and significant consequences to such a compression of the torsional-puckering energy levels. The characteristic torsional-puckering pseudorotation time scale is given by the inverse of the first excitation, which Graf et al. calculated to be $0.56 \mathrm{~cm}^{-1}$ for $\left(\mathrm{D}_{2} \mathrm{O}\right)_{5}$. Consequently, the torsional-puckering motion would be fast compared to the overall rotation of the cluster, leading to pseudorotational averaging of the ten chiral versions of GS into an oblate top with $C_{5 h}$ symmetry on the rotational time scale, regardless of whether the pseudorotation itself were excited. While most other inter- and intermolecular vibrations are much faster than the torsional-puckering motion and can probably be considered to be adiabatically decoupled from this pseudorotation, a few cannot. Notably, Graf et al. indicate that the quasi-pure torsional modes, modes 5, 6, and 7 from Table 2, are likely to couple strongly to the pseudorotation and may even be part of the torsional-puckering energy manifold. ${ }^{31}$ They also note that coupling to pseudorotation by vibrational angular momentum may occur for pairs of normal modes which are degenerate in the limit of pentagonal symmetry, such as modes 5 and 6 . 
TABLE 4: Calculated Intensities (debye ${ }^{2}$ ) and Frequencies $\left(\mathrm{cm}^{-1}\right)$ of Parallel $\left(\mathrm{H}_{2} \mathrm{O}\right)_{5}$ and $\left(\mathrm{D}_{2} \mathrm{O}\right)_{5}$ Torsional-Puckering Pseudorotational Bands ${ }^{a}$

\begin{tabular}{cccccc}
\hline & \multicolumn{2}{c}{$\left(\mathrm{H}_{2} \mathrm{O}\right)_{5}$} & & \multicolumn{2}{c}{$\left(\mathrm{D}_{2} \mathrm{O}\right)_{5}$} \\
\cline { 2 - 3 } \cline { 5 - 6 } band & frequency & intensity & & frequency & intensity \\
\hline $2^{0} \rightarrow 3^{0}$ & 5.3 & 1.01 & & 2.3 & 1.22 \\
$1^{0} \rightarrow 4^{0}$ & 14.8 & 1.09 & & 6.1 & 1.26 \\
$0^{0} \rightarrow 5^{0}$ & 19.1 & 0.59 & & 7.6 & 0.65 \\
$5^{0} \rightarrow 5^{1}$ & $\mathbf{3 9 . 0}^{b}$ & & & $\mathbf{3 7 . 7}^{b}$ & \\
$0^{0} \rightarrow 5^{1}$ & $\mathbf{5 8 . 0 9}^{b}$ & & & $\mathbf{4 5 . 3 7}^{b}$ & \\
$1^{0} \rightarrow 4^{1}$ & 62.6 & 0.09 & & 47.4 & 0.04 \\
$2^{0} \rightarrow 3^{1}$ & 73.5 & 0.2 & & 52.7 & 0.05 \\
$5^{1} \rightarrow 0^{1}$ & 80.3 & 0.28 & & 39.5 & 0.32 \\
$3^{0} \rightarrow 2^{1}$ & 87 & 0.24 & & 60.4 & 0.13 \\
$4^{0} \rightarrow 1^{1}$ & 103.2 & 0.2 & & 69.9 & 0.09 \\
$5^{0} \rightarrow 0^{1}$ & $\mathbf{1 1 9 . 3 2}$ & & & $\mathbf{7 7 . 2 4}$ & \\
$0^{0} \rightarrow 0^{1}$ & $\mathbf{1 3 8 . 4 1}^{b}$ & & & $\mathbf{8 4 . 8 7 ^ { b }}$ & \\
$5^{0} \rightarrow 0^{2}$ & 134.4 & 0.14 & & 95 & 0.09 \\
$0^{0} \rightarrow 0^{2}$ & $\mathbf{1 5 3 . 4 7}^{b}$ & & & $\mathbf{1 0 2 . 6 1}$ & \\
$4^{0} \rightarrow 1^{2}$ & 159.8 & 0.08 & & 106.2 & 0.09 \\
$3^{0} \rightarrow 2^{2}$ & 196.9 & 0.02 & & 126 & 0.04 \\
$2^{0} \rightarrow 3^{2}$ & 236.5 & 0.01 & & 148.2 & 0.02 \\
$1^{0} \rightarrow 4^{2}$ & $277.18^{b}$ & & & $171.67^{b}$ &
\end{tabular}

${ }^{a}$ Bands linking nondegenerate states shown in bold. ${ }^{b}$ Intensities not calculated.

Graf et al. used their torsional-puckering pseudorotational model to calculate transition intensities for many of the lowlying pseudorotational transitions and proposed a reassignment of the parallel $81.2 \mathrm{~cm}^{-1}\left(\mathrm{D}_{2} \mathrm{O}\right)_{5}$ band of Liu et al. Their calculations concerning parallel bands are reproduced in Table 4 along with the frequencies of some additional bands that they did not report. Graf et al. limited their search to bands linking nondegenerate states (in bold), in accord with the conclusion of Liu et al. based on the lack of Coriolis perturbations in the band, and to bands originating from the ground state based on the insensitivity of the measured spectra to heating of the sample reservoir. They also noted that the small observed change in the $B$ rotational constant (viz., $0.35 \mathrm{MHz}$ ) argued against a framework intermolecular mode, again in accord with Liu et al. The only two normal modes with calculated harmonic frequencies close to $81.2 \mathrm{~cm}^{-1}$ are numbers 3 and 4 from Table 2 , but since these vibrations are $x / y$ translational framework modes, they would give rise to perpendicular bands and so were ruled out. The next closest normal modes, numbered 5 and 6 , lie at considerably higher frequencies (viz., 141.4 and 145.8 $\mathrm{cm}^{-1}$ ) but, given their torsional character, are expected to couple into the higher part of the torsional-puckering manifold, and Graf et al. argued that they would therefore not be expected to lie near the calculated harmonic frequencies. We remark nonetheless that harmonic models of intermolecular vibrations poorly describe the floppy motions of water clusters.

Thus, the 1-D torsional-puckering pseudorotational model was used with the knowledge that an $81.2 \mathrm{~cm}^{-1}$ transition frequency would give rise to a dimensionless transition energy $b=3.926$ on the generalized torsional-puckering energy level diagram (Figure 7). Graf et al. examined zero-, high-, and intermediatebarrier heights with regard to this energy. In all of the limits, the $k=5^{0} \leftarrow 0^{0}$ transition is expected to lie at a very low frequency and was not further investigated. In the zero-barrier limit, the $k=0^{1} \leftarrow 0^{0}$ transition frequency is close to the experimentally deduced $b$ value. Unfortunately the $k=0^{1}$ and $0^{2}$ states are degenerate, in discord with the experimental findings, since Coriolis coupling would be expected to split these degeneracies to produce a dedoubling of the bands, whereas only one solitary band was observed. In the high-barrier limit, the $k=5^{1} \leftarrow 0^{0}$ transition proposed by Liu et al. seemed promising and would have resulted in a barrier of $s=8.096$. However, Graf et al. ruled this assignment out on the basis of the low expected intensity outside of the low-barrier limit and the fact that bands predicted to be more intense had not yet been observed. Although the $30.2 \mathrm{~cm}^{-1}$ band was not considered by these authors, we calculate that $b$ would equal 1.46 for this band and does not correspond to any feasible transition, based on Figure 7, in the high-barrier limit, supporting Graf's conclusion. The $89 \mathrm{~cm}^{-1}$ Coriolis perturbed band of Brown et al. ${ }^{12}$ was not considered by Graf et al. either. This band could be attributed to any number of transitions that involve at least one degenerate level and simultaneously fulfill the overall torsional-puckering pseudorotational selection rules for a parallel band. The calculated dimensionless transition energy for this band would be $b=2.57$, and one would expect a barrier lower than that of $\left(\mathrm{D}_{2} \mathrm{O}\right)_{5}$, although not by a full factor of 2 because of the heavy oxygen framework puckering motion. Clearly, in the high barrier limit, there is no feasible match for this transition. With an intermediate barrier height, however, Graf et al. were able to match the $81.2 \mathrm{~cm}^{-1}$ band to either the $k=$ $0^{1} \leftarrow 5^{0}$ or the $k=0^{2} \leftarrow 5^{0}$ transition. While the predicted intensity of the former is quite low, that of the latter is predicted to be high, $\left|\mu_{z}\right|^{2}=1.47 \times 10^{-6}$ and $0.124 \mathrm{D}^{2}$, respectively (reported value differs from reported value in original table because of change in barrier height). Graf et al. therefore opted for the $k=0^{2} \leftarrow 5^{0}$ assignment. We can revisit the $30.2 \mathrm{~cm}^{-1}$ band assignment, this time in the intermediate barrier limit of $s=2.757$. This transition then coincides well with $k=5^{1} \leftarrow$ $5^{0}$. Unfortunately, its predicted intensity was not calculated. The $89 \mathrm{~cm}^{-1}\left(\mathrm{H}_{2} \mathrm{O}\right)_{5}$ band also matches with the predicted $k=3^{1}$ $\leftarrow 2^{0}$ transition, which is reported to be quite strong at $0.2 \mathrm{D}^{2}$ (Table 4), although as previously stated, Graf et al. failed to mention this band. They did state, however, that bands were expected at $9.96 \mathrm{~cm}^{-1}, k=5^{0} \leftarrow 0^{0}$, and at $38.2 \mathrm{~cm}^{-1}, k=5^{1}$ $\leftarrow 0^{0}$, with intensities of 0.608 and $2.48 \times 10^{-6} \mathrm{D}^{2}$, respectively. In fact, many other experimentally observable bands are expected if the first torsional energy manifold is indeed highly compacted, since even at the low temperatures of our cluster source, states up through $5^{0}$ can be expected to be thermally populated, at least for $\left(\mathrm{D}_{2} \mathrm{O}\right)_{5}$. Clearly, additional experimental data are required to unambiguously establish the energies of the torsional-puckering levels.

\section{Experimental Section}

A. Berkeley Terahertz Spectrometers. The Berkeley Terahertz VRT spectrometers have been described in detail elsewhere. ${ }^{36,37}$ Therefore, only those details relevant to the present experiments will be reported here. Rovibrationally cooled $\mathrm{D}_{2} \mathrm{O}$ clusters were formed by bubbling $\mathrm{Ar}$ or $\mathrm{Ne}$ through a reservoir of room-temperature $\mathrm{D}_{2} \mathrm{O}$ (1) (Cambridge Isotopes, 99.9\%) and subsequently adiabatically expanding the saturated $\left(\sim 1 \% \mathrm{D}_{2} \mathrm{O}\right)$ carrier gas through a 10-cm-long, pulsed, planar slit nozzle designed by Liu et al. ${ }^{38}$ into a high-throughput vacuum chamber. Terahertz radiation generated through the first-order mixing of tunable microwave radiation with the output of a fixed frequency far-infrared (FIR) molecular gas laser was multipassed through the expansion 18-20 times in a Herriot cell configuration. ${ }^{39}$ For the lower frequency $\left(\mathrm{D}_{2} \mathrm{O}\right)_{5}$ band at $27.3 \mathrm{~cm}^{-1}$, three FIR lasers were used: $761.6083 \mathrm{GHz}(\mathrm{HCOOH}), 787.7555 \mathrm{GHz}$ (DCOOD), and $849.8280 \mathrm{GHz}$ (HCOOD). Only two FIR lasers, $1397.1186 \mathrm{GHz}\left(\mathrm{CH}_{2} \mathrm{~F}_{2}\right)$ and $1530.8499 \mathrm{GHz}\left(\mathrm{CH}_{2} \mathrm{~F}_{2}\right)$, were needed, however, to scan the majority of the four high-frequency $\left(\mathrm{D}_{2} \mathrm{O}\right)_{5}$ bands; a third laser at $1626.0626 \mathrm{GHz}\left(\mathrm{CH}_{2} \mathrm{~F}_{2}\right)$ was used for the highest-frequency transitions of the $50.7 \mathrm{~cm}^{-1}$. Such a 


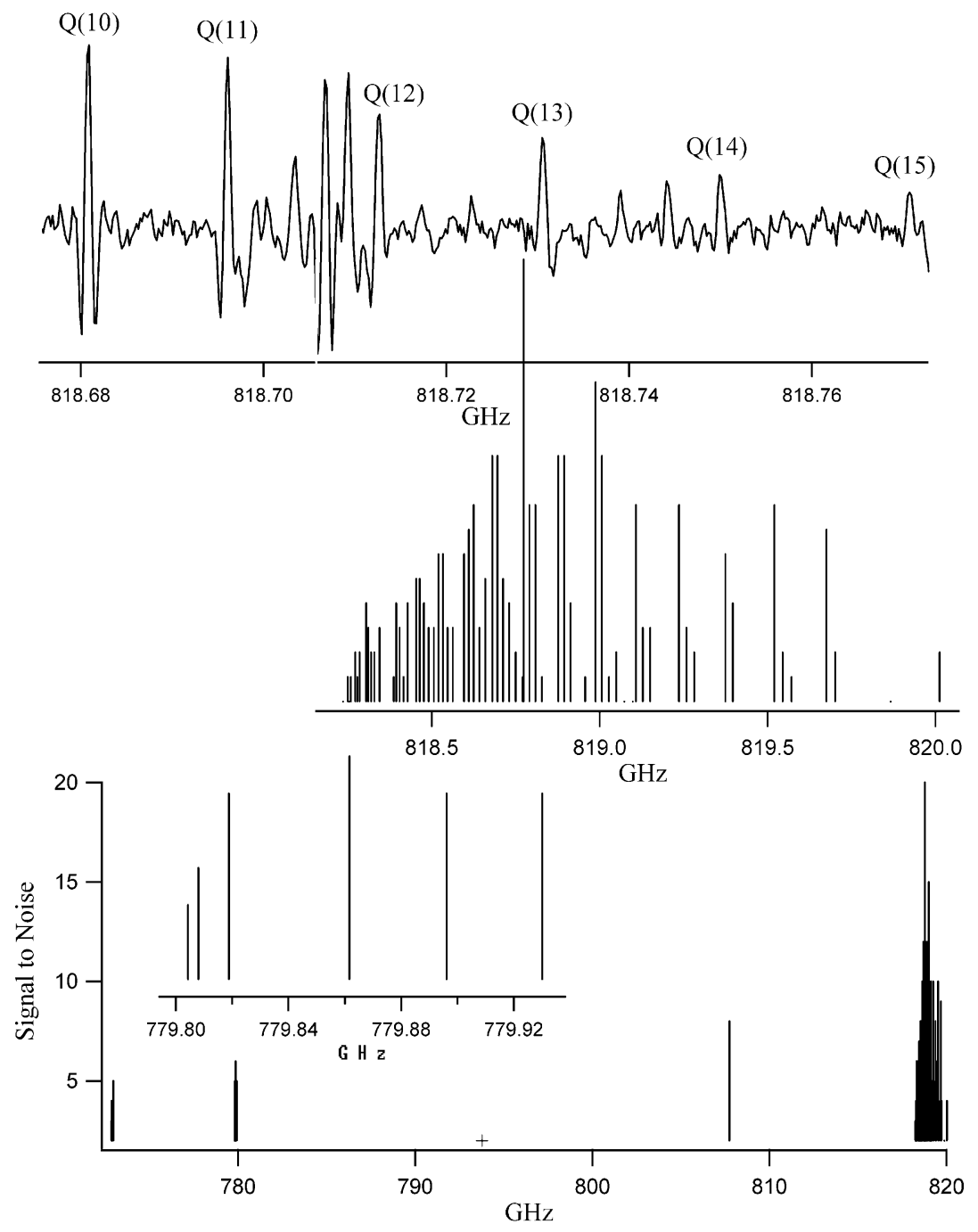

Figure 8. $27.3 \mathrm{~cm}^{-1}(818.2311 \mathrm{GHz})$ band of $\left(\mathrm{D}_{2} \mathrm{O}\right)_{5}$. The bottom graph shows a stick spectrum of the 82 observed transitions for this parallel band, the middle graph expanded views of the Q-branch region and the $\mathrm{P}(11)$ transitions, and the top graph an expanded view of the actual scans of $\mathrm{Q}(10)-\mathrm{Q}(15)$ of the $K=10$ stack. Note: The sole $\mathrm{P}(7)$ transition at $793.767 \mathrm{GHz}$ is tagged so as to be observable.

high band density arising from a single species is quite rare and has interesting implications for the torsional energy manifold that will be discussed below. Transient absorption signals were then measured using a variable-field Putley-mode (VFP) photoconducting InSb detector (Advanced Kinetics). By varying the current in the superconducting solenoid that encircles the InSb crystal, the responsivity profile for the detector can be tuned to detect terahertz radiation from 10 to $80 \mathrm{~cm}^{-1}$. After the $50 \mathrm{kHz}$ modulated terahertz radiation had been detected by the VFP, it was amplified using an Infrared Labs (model ULN6, series 2302) preamplifier, filtered using a Stanford Research Systems (model SR235) low-noise preamplifier, and then collected at $2 f(100 \mathrm{kHz})$ by a lock-in amplifier (SRS-510). The demodulated waveform was then sent to a $100 \mathrm{MHz} / 500 \mathrm{MS} / \mathrm{s}$ Tektronix TDS-320 digital storage oscilloscope capable of rapidly averaging the entire $2.5 \mathrm{~ms}$ full-width at half-maximum (fwhm) wide signal of a typical absorption event over 32 or 64 nozzle pulses. The background (taken when the pulsed nozzle is off)-subtracted average of the digitized waveform was then transferred to a PC for long-term display and storage purposes. It should be noted that, although the spectral resolution of the spectrometer is determined by Doppler broadening, FIR laser drift results in an absolute spectral accuracy of about $3 \mathrm{MHz}$.

B. 27.3 and $50.7 \mathbf{~ c m}^{-1}$ Bands. The stick spectra of the two unperturbed $\left(\mathrm{D}_{2} \mathrm{O}\right)_{5}$ parallel bands measured at $27.3(818.2311$
$\mathrm{GHz})$ and $50.7 \mathrm{~cm}^{-1}(1521.3443 \mathrm{GHz})$ are shown in Figures 8 and 9 , respectively. The $27.3 \mathrm{~cm}^{-1}$ band is by far the weakest and most sparse of all the currently observed $\left(\mathrm{D}_{2} \mathrm{O}\right)_{5}$ bands. In addition, this pentamer band was impropitiously located under the much stronger, $100: 1, \mathrm{D}_{2} \mathrm{O}$ trimer band centered at 28.0 $\mathrm{cm}^{-1}$. A total of 82 transitions, compared to 244 for the 50.7 $\mathrm{cm}^{-1}$ band, were fit to an oblate symmetric rotor parallel $(\Delta K$ $=0$ ) band. The dense nature of the Q-branch region along with the clumping of transitions into $K$-stacks is shown in the middle of Figures 8 and 9, along with an additional magnification of the $\mathrm{P}(11)$ transitions for the $27.3 \mathrm{~cm}^{-1}$ band in Figure 8 . The upper graph shows $\mathrm{Q}(10)-\mathrm{Q}(15)$ of the $K=10$ stack and $\mathrm{Q}(14)-\mathrm{Q}(18)$ of the $K=14$ stack in Figures 8 and 9, respectively. The stronger Q-branch lines have signal-to-noise ratios on the order of $12-20: 1$ and $30-40: 1$ for the 27.3 and $50.7 \mathrm{~cm}^{-1}$ bands, respectively. The Doppler-limited line widths of these transitions are $\sim 700 \mathrm{kHz}$ fwhm, and none of the transitions display splittings due to bifurcation tunneling, consistent with previously observed $\left(\mathrm{D}_{2} \mathrm{O}\right)_{5}$ bands. Isotope experiments yielded a cluster size of $4.81 \pm 0.27$ at the $95 \%$ confidence level for the $50.7 \mathrm{~cm}^{-1}$ band, while the precise value for the $27.3 \mathrm{~cm}^{-1}$ band was not determined. These transitions were observed in both Ar and Ne carrier gases, indicating that these rare-gas species were not constituents of the absorber. These two bands are very similar to the non-Coriolis perturbed 


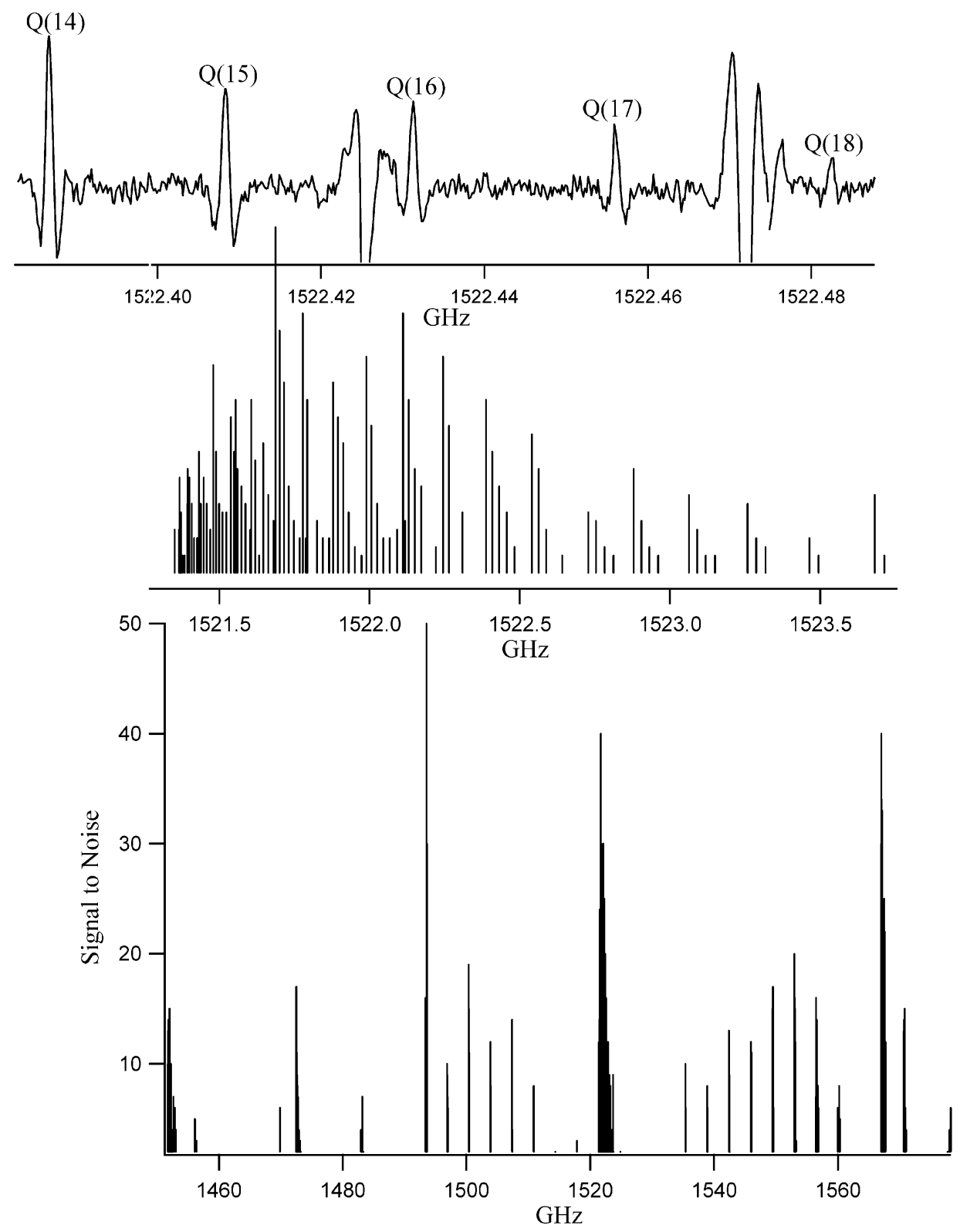

Figure 9. $50.7 \mathrm{~cm}^{-1}(1521.3443 \mathrm{GHz})$ band of $\left(\mathrm{D}_{2} \mathrm{O}\right)_{5}$. The bottom graph shows a stick spectrum of the 244 observed transitions for this parallel band, the middle graph an expanded view of the Q-branch region, and the top graph an expanded view of the actual scans of Q(14)-Q(18) of the $K=14$ stack. Note: Inverted "peaks" are artifacts from Etalon fringes occurring between multipass mirrors.

$\left(\mathrm{D}_{2} \mathrm{O}\right)_{5}$ bands of Liu et al. at $30.2 \mathrm{~cm}^{-1}$ and Cruzan et al. at $81.2 \mathrm{~cm}^{-1}$ and represent the third and fourth observed $\left(\mathrm{D}_{2} \mathrm{O}\right)_{5}$ bands interconnecting nondegenerate torsional-puckering energy levels.

C. 45.0, 45.4, and $47.7 \mathrm{~cm}^{-1}$ Bands. The stick spectra of the $3\left(\mathrm{D}_{2} \mathrm{O}\right)_{5}$ parallel bands measured at $45.0,45.4$, and 47.7 $\mathrm{cm}^{-1}$ are shown in Figures 10, 11, and 12, respectively. The $45.0 \mathrm{~cm}^{-1}(1350.6625 \mathrm{GHz})$ band comprises only 112 assigned transitions compared to 398 and 208 for the 45.4 , and $47.7 \mathrm{~cm}^{-1}$ bands, respectively. Furthermore, despite its easily recognizable Q-branch, with the strongest transition signal-to-noise ratio $(S /$ $N$ ) being $18: 1$, only $18 / 112$ transitions had a measured $S / N$ greater than 10:1! For comparison, the strongest Q-branch lines of the 45.4 , and $47.7 \mathrm{~cm}^{-1}$ bands, respectively, were measured at $130: 1$ and 50:1, and they had 274/398 and 85/208 transitions with $S / N$ greater than 10:1. It should be noted, however, that the lack of additional P-branch transitions for the $45.0 \mathrm{~cm}^{-1}$ band cannot be entirely attributed to weak transitions, since the lack of spectral coverage prevented scanning of this frequency region below $1337.7 \mathrm{GHz}$. The Doppler-limited line widths of these transitions are $\sim 700 \mathrm{kHz}$ fwhm, and none of the transitions display splittings due to bifurcation tunneling, consistent with previously observed $\left(\mathrm{D}_{2} \mathrm{O}\right)_{5}$ bands. While carrier-gas experiments indicated that the absorbing species did not contain raregas species, and isotope experiments along with the relative spacing between groups of $\mathrm{P}$ - and R-transitions $(\sim 2 B)$ indicated that these 3 bands were due to $\left(\mathrm{D}_{2} \mathrm{O}\right)_{5}$, the overall band profiles were markedly different from those of previously observed $\left(\mathrm{D}_{2} \mathrm{O}\right)_{5}$ bands. Nonetheless, these 3 bands were found to be quite similar in structure not only to one another but also to the 89 $\mathrm{cm}^{-1}$ Coriolis perturbed $\left(\mathrm{H}_{2} \mathrm{O}\right)_{5}$ band reported by Brown et al. ${ }^{12}$ The middle traces in Figures 10, 11, and 12 show expanded views of the Q-branch regions of these 3 new bands. Each Q-branch has been split into two distinct groups of transitions; the accidental degeneracy of $k= \pm n$ levels has been effectively lifted by Coriolis coupling. The high-frequency sides appear 


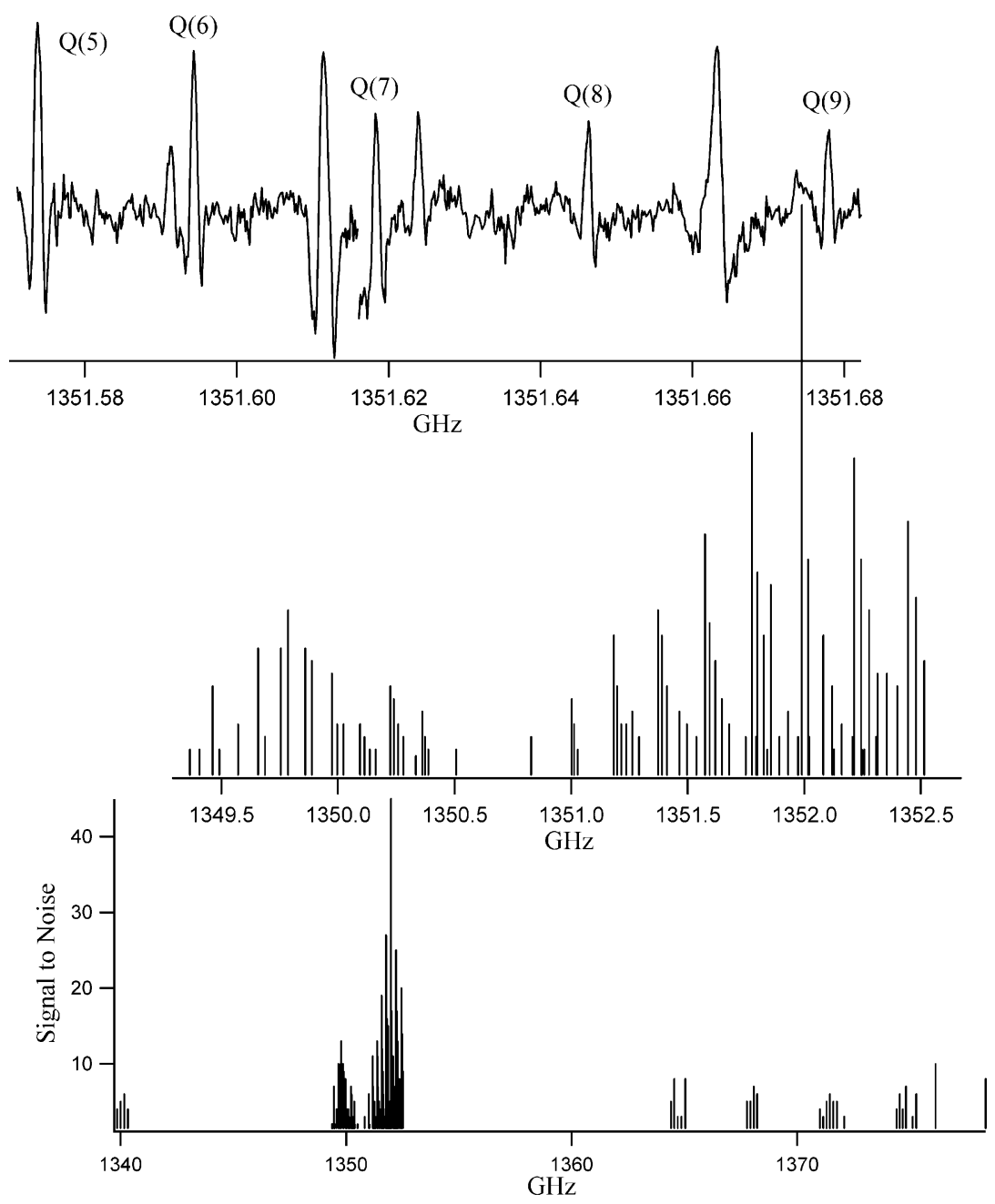

Figure 10. $45.0 \mathrm{~cm}^{-1}(1350.6625 \mathrm{GHz})$ band of $\left(\mathrm{D}_{2} \mathrm{O}\right)_{5}$. The bottom graph shows a stick spectrum of the 112 observed transitions for this parallel band, the middle graph an expanded view of the repelling Q-branch region, and the top graph an expanded view of the actual scans of Q(5)-Q(9) of the $K=5$ stack.

very similar to the non-Coriolis perturbed Q-branches of the $30.2,50.7$, and $81.2 \mathrm{~cm}^{-1}$ bands, with transitions grouped into $K$-stacks and straightforward to assign. The low-frequency sides, however, appear highly perturbed and proved more challenging to assign because of the less obvious clumping of transitions and to the higher line density. It is interesting to note that the splitting effect of the Coriolis coupling increases from the least affected band at $47.7 \mathrm{~cm}^{-1}$ to the most affected band at 45.0 $\mathrm{cm}^{-1}$.

\section{Results and Analysis}

A. 27.3 and $\mathbf{5 0 . 7} \mathbf{c m}^{-1}$ Bands. Considering the similarities between the 27.3 and $50.7 \mathrm{~cm}^{-1}$ bands and the two previously measured $\left(\mathrm{D}_{2} \mathrm{O}\right)_{5}$ bands, 82 and 244 transitions, respectively, were assigned and fit (Table VS and VIS in Supporting Information) to the same non-Coriolis perturbed energy level expression for an oblate, symmetric rotor

$$
\begin{array}{r}
E_{J, K}^{v}=v+B J(J+1)+(C-B) K^{2}-D_{J} J^{2}(J+1)^{2}- \\
D_{J, K} J(J+1) K^{2}
\end{array}
$$

where $v$ is the vibrational energy, $J$ the rotational quantum number, $K$ the projection of $J$ along the $z$ axis, $B$ and $C$ the rotational constants, and $D_{J}$ and $D_{J, K}$ are the quartic distortion constants. The resulting rotational parameters are listed in Table 5 along with those for the 30.2 and $81.2 \mathrm{~cm}^{-1}$ bands. The root- mean-square (rms) of the fit residuals (observed frequency minus the frequency calculated from the fitted parameters) was 0.63 $\mathrm{MHz}$ for the $27.3 \mathrm{~cm}^{-1}$ band and $0.86 \mathrm{MHz}$ for the $50.7 \mathrm{~cm}^{-1}$ band, well within both the absolute experimental accuracy of $\sim 3 \mathrm{MHz}$ and the spectral resolution of $1 \mathrm{MHz}$.

As with any parallel symmetric top band, independent determination of the $C$ rotational constants is not possible, since this requires the measurement of a perpendicular band. Therefore, only the difference between the upper- and lower-state $C$ rotational constants could be fit (viz., $\Delta C=C^{\prime}-C^{\prime \prime}$ ). Furthermore, in his fit of the $30.2 \mathrm{~cm}^{-1}$ band, Cruzan set the excited- and ground-state distortion parameters $\left(D_{J}\right.$ and $\left.D_{J, K}\right)$ equal because of large correlations between them. Although the same correlations were observed in the 27.3, 50.7, and 81.2 $\mathrm{cm}^{-1}$ bands, their distortion parameters were fit independently (Table VIIIS in Supporting Information). Additionally, inspection of the correlation matrices reveals a high correlation between $B^{\prime}$ and $B^{\prime \prime}$ for all four fits. Care must therefore be taken when using any of these highly correlated parameters for spectral interpretation and comparison.

It is tempting, given that the ground-state $B$ rotational constants of all three bands deviate by less than $1 \mathrm{MHz}$, to conclude that these bands originate in the same ground state. However, applying similar considerations to the excited state, one finds that the 30.2 and $81.2 \mathrm{~cm}^{-1}$ bands have excited-state $B$ rotational constants that vary by even less (viz., less than 0.1 

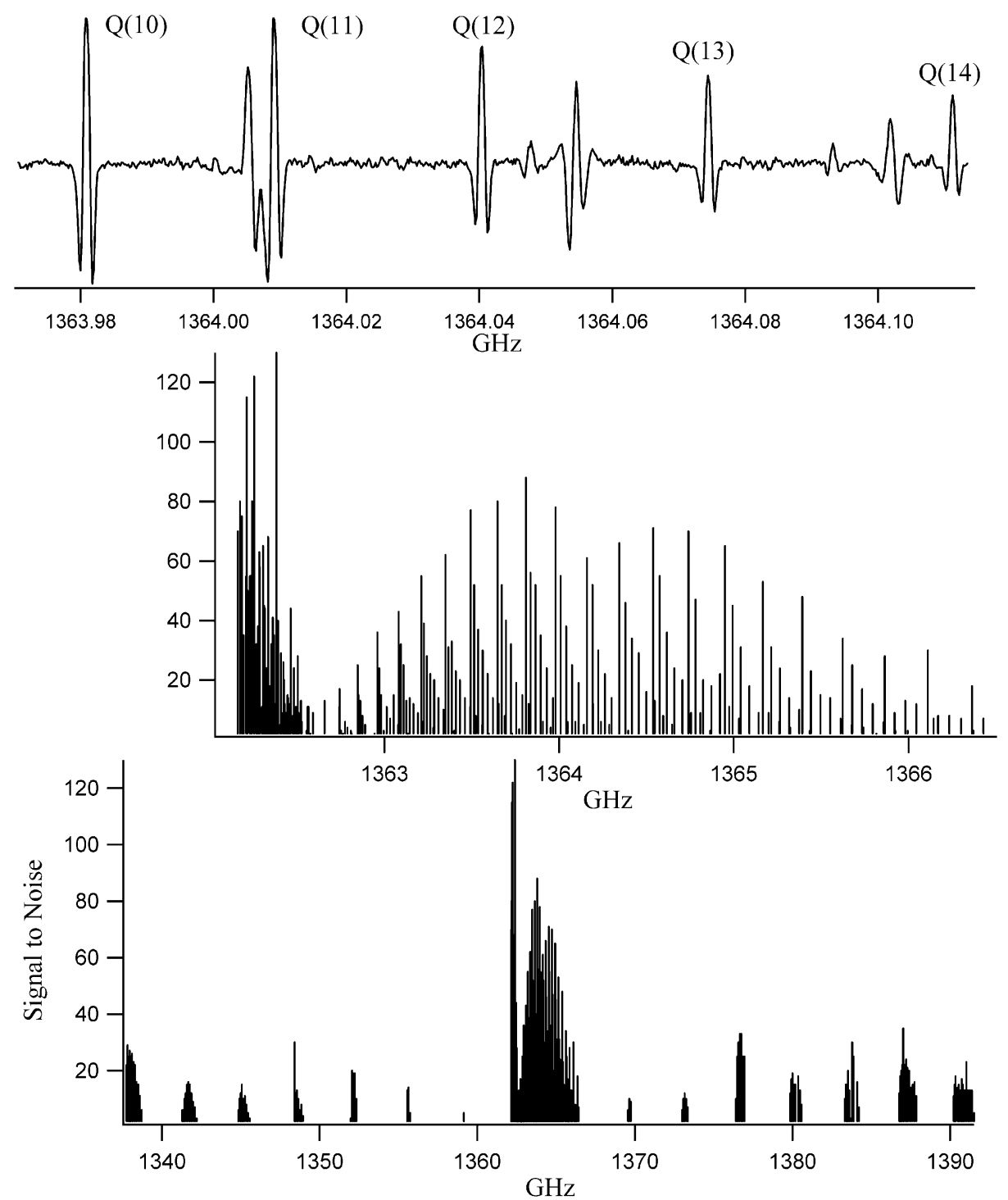

Figure 11. $45.4 \mathrm{~cm}^{-1}(1362.6412 \mathrm{GHz})$ band of $\left(\mathrm{D}_{2} \mathrm{O}\right)_{5}$. The bottom graph shows a stick spectrum of the 398 observed transitions for this parallel band, the middle graph an expanded view of the repelling Q-branch region, and the top graph an expanded view of the actual scans of Q(10)$\mathrm{Q}(14)$ of the $K=10$ stack.

$\mathrm{MHz})$ ! Given the low vibrational and rotational temperatures of our cluster expansion, it is unlikely that these two bands terminate in the same state, which would require the initial state of the $30.2 \mathrm{~cm}^{-1}$ band to lie over $50 \mathrm{~cm}^{-1}$ above the ground state (the $42.9 \mathrm{~cm}^{-1}\left(\mathrm{H}_{2} \mathrm{O}\right)_{3}$ band that originates at $22.7 \mathrm{~cm}^{-1}$ above the ground state is one of the highest documented to date). Overall, the rotational parameters of all three bands agree well, thereby allowing confident identification of the 27.3 and 50.7 $\mathrm{cm}^{-1}$ bands as new, non-Coriolis perturbed $\left(\mathrm{D}_{2} \mathrm{O}\right)_{5}$ bands.

B. 45.0, 45.4, and $47.7 \mathrm{~cm}^{-1}$ Bands. The 45.0, 45.4, and $47.7 \mathrm{~cm}^{-1}$ bands all show strong Coriolis perturbations. Figure 13 depicts one such effect through comparison of the $R(6)$ transitions of the unperturbed $50.7 \mathrm{~cm}^{-1}$ band and the $\mathrm{R}(6)$ transitions of the Coriolis-perturbed $45.4 \mathrm{~cm}^{-1}$ band. The 50.7 $\mathrm{cm}^{-1}$ band exhibits the $(J+1)$ transitions expected of a P- or $\mathrm{R}$-branch corresponding to an unperturbed oblate symmetric top and shows a constant second difference in the spacing between successive transitions. Rather than $(J+1)$ transitions, the 45.4 $\mathrm{cm}^{-1}$ band, like the 45.0 and $47.7 \mathrm{~cm}^{-1}$ bands, exhibits $(2 J+$ 1) transitions within the P- and R-branches. Despite the extra transitions, a constant second difference is maintained. By cataloging the transitions from higher $J$ to lower $J$, it is clear that each $K>0$ transition is split into two, one on either side of the solitary $K=0$ transition. Furthermore, while the magnitude of the splitting increases with $K$, for a given $K$, the splitting remains constant regardless of $J$. For example, the $K$ $=1 \mathrm{R}$-branch transitions are split into two, separated by 183 $\mathrm{MHz}$, while all $K=6 \mathrm{R}$-branch transition pairs, regardless of $J$, are split by a greater, yet constant amount (viz., $1096 \mathrm{GHz}$ ). The magnitude of the observed splitting in the P- and R-branches of these three bands is therefore clearly independent of $J$ and increases in a regular manner with $K$. Moreover, the splitting is linearly dependent on $K$ for all three of these Coriolis perturbed bands. The linear dependence of the splitting on $K$ is consistent with the presence of first-order Coriolis coupling.

Second-order Coriolis effects observed in the $28 \mathrm{~cm}^{-1}\left(\mathrm{D}_{2} \mathrm{O}\right)_{3}$ band by Viant et al. ${ }^{29}$ are manifested as relatively large $(\sim 17$ $\mathrm{MHz}$ ) errors in the fit residuals for high $J$ transitions in particular $K$ states ( $K=2$ in the case of this particular trimer band) relative to the other $K$ states. Because of the similarities in structure and dynamics between the trimer and the pentamer, it is reasonable to expect second-order Coriolis coupling to occur in the pentamer. However, inspection of the residuals in Tables IXS, XS, and XIS (Supporting Information) indicate that, in accord with Brown's $\left(\mathrm{H}_{2} \mathrm{O}\right)_{5}$ band, only first-order Coriolis coupling is present in the pentamer. Therefore, while it is likely 


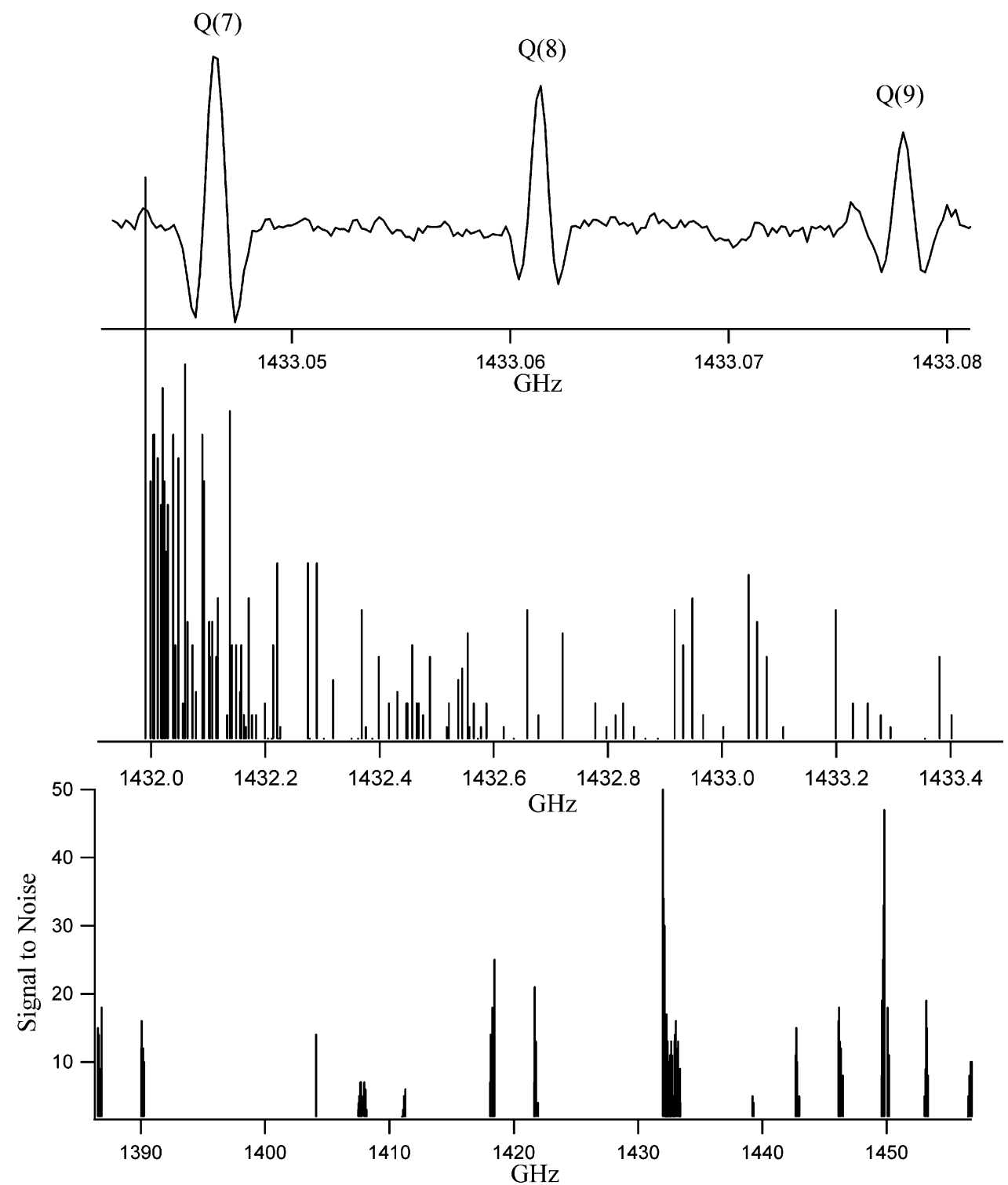

Figure 12. $47.7 \mathrm{~cm}^{-1}(1432.2700 \mathrm{GHz})$ band of $\left(\mathrm{D}_{2} \mathrm{O}\right)_{5}$. The bottom graph shows a stick spectrum of the 208 observed transitions for this parallel band, the middle graph an expanded view of the repelling Q-branch region, and the top graph an expanded view of the actual scans of Q(7)-Q(9) of the $K=7$ stack.

TABLE 5: Spectroscopic Parameters (MHz) by Band as Determined via Least-Squares Analysis ${ }^{a}$

\begin{tabular}{lllll}
\hline & \multicolumn{1}{c}{$27.3 \mathrm{~cm}^{-1}$} & \multicolumn{1}{c}{$50.7 \mathrm{~cm}^{-1}$} & \multicolumn{1}{c}{$30.2 \mathrm{~cm}^{-1 b}$} & \multicolumn{1}{c}{$81.2 \mathrm{~cm}^{-1} c$} \\
\hline$B^{\prime \prime}$ & $1750.87(4)$ & $1751.014(8)$ & $1750.964(2)$ & $1750.815(8)$ \\
$D_{J^{\prime \prime}}$ & $0.0031(1)$ & $0.00364(2)$ & $0.00324(2)$ & $0.00159(5)$ \\
$\mathrm{D}_{J^{\prime \prime}, K^{\prime \prime}}$ & $-0.0077(7)$ & $-0.00648(6)$ & $-0.00691(6)$ & $-0.0048(2)$ \\
$\nu_{\mathrm{o}}$ & $818231.2(2)$ & $1521344.3(1)$ & $905368.415(9)$ & $2434074.36(6)$ \\
$B^{\prime}$ & $1751.57(4)$ & $1751.738(8)$ & $1751.116(2)$ & $1751.163(8)$ \\
$D_{J^{\prime}}$ & $0.0031(1)$ & $0.00359(3)$ & $=\mathrm{D}_{\mathrm{J}^{\prime \prime}}$ & $0.00163(5)$ \\
$D_{J^{\prime}, K^{\prime}}$ & $-0.0077(7)$ & $-0.00641(6)$ & $=\mathrm{D}_{J^{\prime \prime}, \mathrm{K}^{\prime \prime}}$ & $-0.0048(2)$ \\
$\Delta C$ & $4.428(3)$ & $5.267(2)$ & $2.42156(9)$ & $5.267(2)$ \\
$\sigma_{\mathrm{rms}}$ & 0.63 & 0.86 & 0.56 & 3.4
\end{tabular}

${ }^{a} 1 \sigma$ uncertainty of last significant digit in parentheses. ${ }^{b}$ ref $11 .{ }^{c}$ ref 13 .

that a second-order Coriolis effect does occur in the water pentamer, there is no definitive signature of its presence in any of the four Coriolis perturbed pentamer bands, and thus, this effect was not included in the final fits of the 45.0, 45.4, and $47.7 \mathrm{~cm}^{-1}$ bands.

The oblate symmetric top energy expression given in eq 1 was modified according to Teller ${ }^{40}$ to include the first-order Coriolis coupling term $\pm 2 \zeta C$, where $\zeta$ is the coupling constant and $C$ is the $B_{z}$ rotational constant for an oblate rotor. The minus sign applies if the vibrational angular momentum has the same direction as the rotational angular momentum, whereas the plus sign applies if they are in opposing directions

$$
\begin{array}{r}
E_{J, K}^{v}=v+B J(J+1)+(C-B) K^{2}-D_{J} J^{2}(J+1)^{2}- \\
D_{J, K} J(J+1) K^{2}-D_{K} K^{4} \pm 2 \zeta C K
\end{array}
$$

The resulting rotational parameters are listed in Table 6 . The rms values of the fit residuals are 1.49, 1.80, and 1.61 MHz for the $45.0,45.4$, and $47.7 \mathrm{~cm}^{-1}$ bands, respectively, well within the experimental error of $\sim 3 \mathrm{MHz}$ yet slightly greater than the spectral resolution of $1 \mathrm{MHz}$. Correlation matrices for these three bands can be found in Table XIIIS (Supporting Information).

While it is difficult to compare the rms values for the unperturbed bands among one another, given that they span a large frequency region and therefore have very different spectral resolutions and intensities due to the relative strength of the FIR lasers used, it is quite possible to compare the 45.0 and $45.4 \mathrm{~cm}^{-1}$ bands, since they overlap in frequency. The 45.0 $\mathrm{cm}^{-1}$ band is the second weakest and most highly perturbed of 


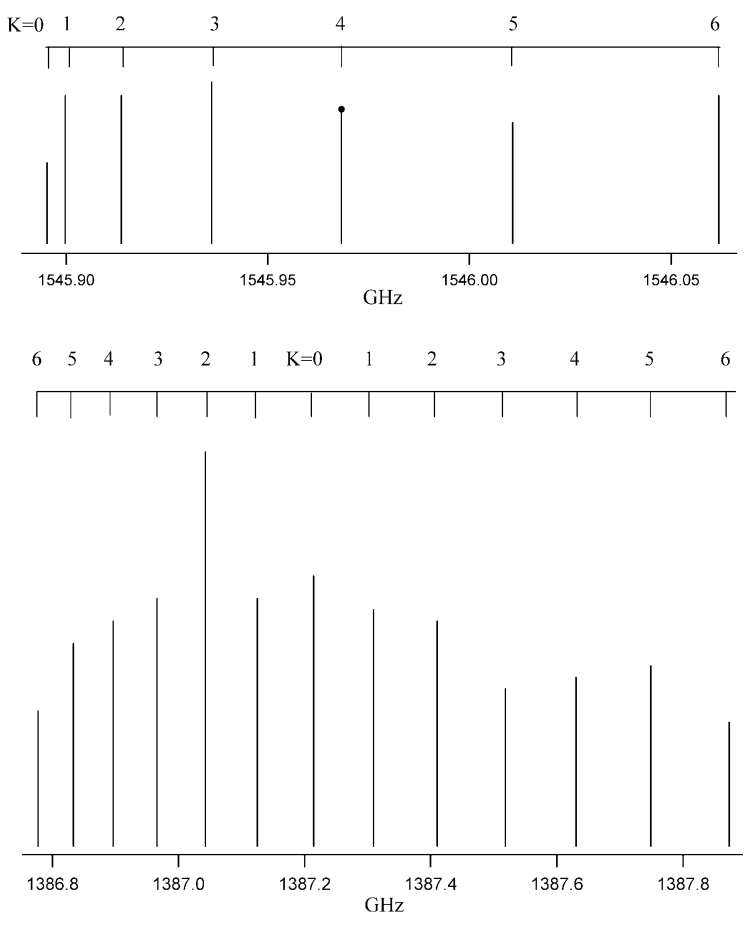

Figure 13. First-order Coriolis effects on $R(6)$ transitions. The top graph shows a stick spectrum of the seven $\mathrm{R}(6)$ transitions of the unperturbed, oblate symmetric rotor band at $50.7 \mathrm{~cm}^{-1}$ (the $K=4$ transition is tagged because it was not observed experimentally). The bottom graph shows a stick spectrum of the thirteen $\mathrm{R}(6)$ transitions of the Coriolis perturbed band at $45.4 \mathrm{~cm}^{-1}$.

TABLE 6: Spectroscopic Parameters $(\mathrm{MHz})^{a}$

\begin{tabular}{llll}
\hline & \multicolumn{1}{c}{$45.0 \mathrm{~cm}^{-1}$} & \multicolumn{1}{c}{$45.4 \mathrm{~cm}^{-1}$} & \multicolumn{1}{c}{$47.7 \mathrm{~cm}^{-1}$} \\
\hline$B^{\prime \prime}$ & $1751.976(77)$ & $1750.859(42)$ & $1751.335(25)$ \\
$D_{J^{\prime \prime}}$ & $0.0019(10)$ & $0.0029(5)$ & $0.0031(1)$ \\
$D_{J^{\prime \prime}, K^{\prime \prime}}$ & $-0.0116(29)$ & $-0.0071(12)$ & $0.0043(4)$ \\
$v_{\mathrm{o}}$ & $1350662.5(4)$ & $1362641.2(2)$ & $1432270.0(3)$ \\
$B^{\prime}$ & $1752.787(76)$ & $1752.055(42)$ & $1752.277(26)$ \\
$D_{J^{\prime}}$ & $0.0017(10)$ & $0.0026(5)$ & $0.0032(2)$ \\
$D_{J^{\prime}, K^{\prime}}$ & $-0.0089(29)$ & $-0.0069(12)$ & $0.0073(4)$ \\
$\Delta D_{K}$ & $-0.0038(3)$ & $0.00046(3)$ & $-0.00050(9)$ \\
$\Delta C$ & $3.99(2)$ & $4.141(3)$ & $5.159(7)$ \\
$\Delta \zeta$ & $20.007(80)$ & $11.072(9)$ & $7.307(9)$ \\
$\sigma_{\text {rms }}$ & 1.49 & 1.80 & 1.61
\end{tabular}

${ }^{a} 1 \sigma$ uncertainty of last significant digit in parentheses.

all the measured $\left(\mathrm{D}_{2} \mathrm{O}\right)_{5}$ bands with only 112 fit transitions, most of which arise from $J \leq 15$. The $45.4 \mathrm{~cm}^{-1}$ band, on the other hand, is the strongest of the perturbed bands, with $398 \mathrm{fit}$ transitions and consistently high $J$ values peaking at $J=25$. The rms values of these two bands, 1.49 and 1.80 respectively, are not only similar, but are also not much greater than those of the unperturbed bands, particularly at comparable frequencies. Arguably, the $50.7 \mathrm{~cm}^{-1}$ band with its $0.86 \mathrm{rms}$ was scanned on a different FIR laser line, but it was of comparable strength. We conclude that Coriolis effects have been properly accounted for with first-order terms, since rms values remain fairly constant regardless of the presence of Coriolis perturbation and the addition of high $J$ transitions. It should be noted that $D_{K}$ and $\zeta$, like the $C$ rotational constant, depend only on $K$ and therefore cannot be determined independently from the fit of a parallel band.

C. $103.8 \mathrm{~cm}^{-1}\left(\mathrm{H}_{2} \mathrm{O}\right)_{5}$ Band. One additional $\mathrm{H}_{2} \mathrm{O}$ pentamer band has been identified. Unfortunately, the exceedingly compact nature of this band, compounded by overlapping and unresolved bifurcation tunneling components, prevents unambiguous fitting of the observed transitions. Therefore, we cannot provide rotational constants and other spectroscopic parameters, but we can make a few general remarks. First, this band is unperturbed and has a band origin of roughly $103.8 \mathrm{~cm}^{-1}$. Second, each rovibrational transition exhibits bifurcation tunneling splittings analogous to those found in the $89 \mathrm{~cm}^{-1}$ band reported by Brown et al. ${ }^{12}$ Again, only four of the tunneling components were observed due to nuclear spin statistics and degeneracies. However, the splitting was twice as large (viz., $\sim 11 \mathrm{MHz}$ compared to $4.8 \mathrm{MHz}$ for the $89 \mathrm{~cm}^{-1}$ band). In total, 409 transitions, including all bifurcation tunneling components, were attributed to this $\mathrm{H}_{2} \mathrm{O}$ pentamer band (Figure 14). We note that measurement of a band exhibiting bifurcation tunneling splittings that involves Coriolis perturbed states is required to determine the dynamics that give rise to the observed bifurcation splitting patterns. Indeed, all three splitting patterns determined by Wales et al. predict four equally spaced transitions for non-Coriolis perturbed states but uniquely split transitions for Coriolis perturbed states. Each of the three splitting patterns is associated with bifurcation tunneling accompanied by a precise number of free hydrogen flips. ${ }^{17-19}$

\section{Discussion}

Although it is difficult to rigorously interpret and compare the fitted parameters of the seven $\left(\mathrm{D}_{2} \mathrm{O}\right)_{5}$ bands, a few general remarks can be made. First, only small changes, $<1.2 \mathrm{MHz}$ $(\sim 0.07 \%)$, were observed in the $B$ rotational constants upon vibrational excitation. Second, all of the ground- and excitedstate $B$ rotational constants are markedly similar. Third, all seven of the observed $\left(\mathrm{D}_{2} \mathrm{O}\right)_{5}$ bands fit well to an oblate symmetric top energy expression. Fourth, only small centrifugal distortion constants were needed to fit all of the bands. These observations are all consistent with a vibrational averaging of the motions of the light deuterium atoms, which results in an effective water pentamer structure with $C_{5 h}$ symmetry, as opposed to the chiral, asymmetric $\left(C_{1}\right)$ minimum-energy structure. Graf et al. ${ }^{31}$ have, however, cautioned against a purely pseudorotational assignment, since this averaging may be "fast" with respect to overall rotation and would affect the rotational parameters even if the torsional-puckering pseudorotational vibration itself were not excited. This is especially important in light of the almost purely torsional motions predicted to lie close in frequency to the observed bands. However, these torsional modes are likely to be strongly coupled to the torsional-puckering pseudorotational motion and may even form a part of the torsional-puckering energy manifold. Thus, it seems reasonable to pursue the torsional-puckering pseudorotational model, but with inclusion of the heavy oxygen framework puckering motion and knowledge that the transition state of the excitation may not be exclusively pseudorotational in nature.

Another important piece of information comes from the fact that none of the observed bands that were tested as "hot bands" through heating of the $\mathrm{D}_{2} \mathrm{O}$ reservoir (all except the 27.3 and $45.0 \mathrm{~cm}^{-1}$ bands, which were not tested) showed any response. Consequently, these pentamer bands most likely originate in low energy, highly populated states. It should be noted however that, while the rotational temperature of our cluster source has been determined through careful comparison of Q-branch transition intensities to be $\sim 3-7 \mathrm{~K}$, the vibrational temperature may not be this cold, as evidenced by the observed $\left(\mathrm{H}_{2} \mathrm{O}\right)_{3}$ torsional band originating $22.7 \mathrm{~cm}^{-1}$ above the ground state. ${ }^{24}$ Brown et al. make no mention of whether the intensity of this $\left(\mathrm{H}_{2} \mathrm{O}\right)_{5}$ band increased upon heating, yet it is the band originating in the highest energy state.

We have also now positively identified and analyzed four non-Coriolis perturbed, parallel $\left(\mathrm{D}_{2} \mathrm{O}\right)_{5}$ bands at frequencies 

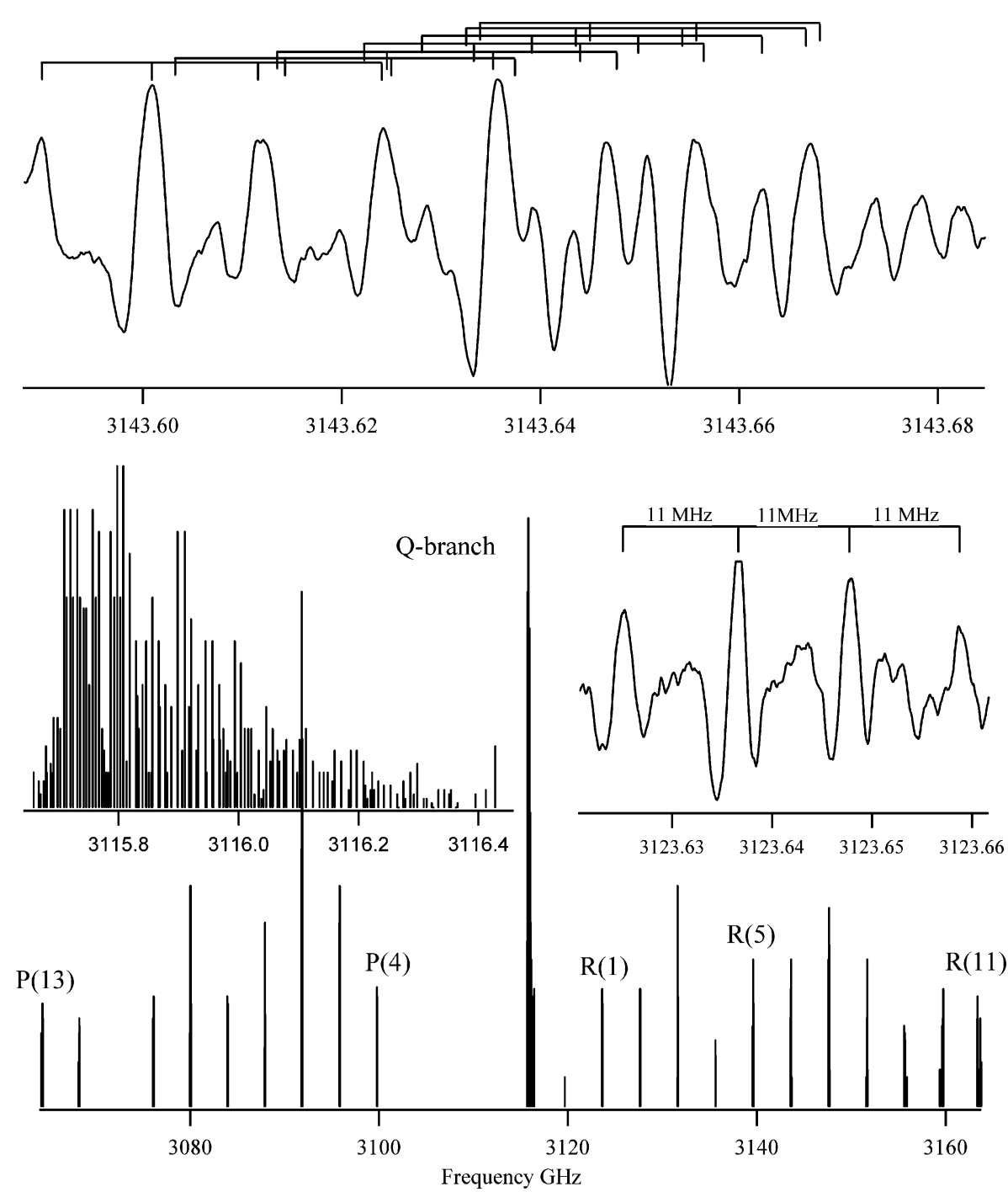

Figure 14. $103.8 \mathrm{~cm}^{-1}\left(\mathrm{H}_{2} \mathrm{O}\right)_{5}$ band. The top graph shows overlapping and unresolved bifurcation tunneling components of $\mathrm{R}(5)$ transitions. The middle left graph provides an expanded view of the dense Q-branch region, whereas the right graph shows the 4 observed bifurcation tunneling components of the $\mathrm{R}(1)$ transition. The bottom graph shows an overview of the 409 observed transitions.

below $82 \mathrm{~cm}^{-1}$. Given that unperturbed bands are expected to arise only from transitions originating and terminating in nondegenerate states and that the overall pseudorotational selection rules, $\Delta K-\Delta k=10 n+5(n=$ integer $)$, set forth by Liu et al. ${ }^{13}$ and accepted by Graf et al., ${ }^{31}$ dictate that only states $k=5$ and 0 will be so linked, a minimum of three torsionalpuckering energy manifolds (ten states each) will be necessary to account for all four observed bands, since the sum of 30.2 or 27.3 and $50.7 \mathrm{~cm}^{-1}$ does not equal $81.2 \mathrm{~cm}^{-1}$. While the resulting sum is actually remarkably close, fitting errors cannot account for the minimum difference of $0.3 \mathrm{~cm}^{-1}(\sim 9 \mathrm{GHz})$, since the experimental uncertainty is on the order of 0.0001 $\mathrm{cm}^{-1}(\sim 3 \mathrm{MHz})$. Moreover, inclusion of the individually reported bands of the water trimer into the global fit resulted in very little change, tens of megahertz at most, of their reported band origins. ${ }^{23}$ The only other scenario within the torsionalpuckering pseudorotational model that could account for four unperturbed, parallel bands under $82 \mathrm{~cm}^{-1}$ within only two torsional manifolds would be to attribute one of the bands to the much weaker $\Delta K= \pm 2$ parallel bands, but also to admit that the Coriolis splitting of the necessarily included degenerate levels was either so small as to be unobservable or of precisely the same magnitude as that of another pair of Coriolis-perturbed levels. Given the three severely Coriolis perturbed bands, 45.0,
45.4 , and $47.7 \mathrm{~cm}^{-1}$, the probability of such a scenario is low. Also, no bands of the $\Delta K= \pm 2$ type were observed in the trimer. It therefore seems reasonable to expect at least three distinct torsional-puckering energy manifolds to be necessary to explain all of the observed transitions.

The next logical step after accepting the idea of requiring at least three different manifolds is to assume a compact lower manifold of states below $\sim 20 \mathrm{~cm}^{-1}$. Indeed, numerous low energy states are necessary to account for the many observed bands. Moreover, given that the bands do not respond as "hot bands" to reservoir heating, it is likely that this lower manifold is even more compact, perhaps on the order of $\sim 5 \mathrm{~cm}^{-1}$, as suggested by Graf et al. ${ }^{31}$ The high density of transitions in a given frequency region supports this proposal. It would not be difficult to account for three Coriolis perturbed bands at 45.0, 45.4 , and $47.7 \mathrm{~cm}^{-1}$ if they all originated from the levels of a compact lower torsional-puckering manifold and terminated in another distinct, compact manifold near $50 \mathrm{~cm}^{-1}$. Furthermore, an unperturbed band was observed at $50.7 \mathrm{~cm}^{-1}$. No transitions have yet been ascribed to the pentamer in the range 30-44.6 $\mathrm{cm}^{-1}$, but searching this region should be made a priority, since a water pentamer transition near $44 \mathrm{~cm}^{-1}$ seems probable. The $81.2 \mathrm{~cm}^{-1}$ band would also be accounted for in this scenario, since it need only be placed alone in a higher-energy manifold. 


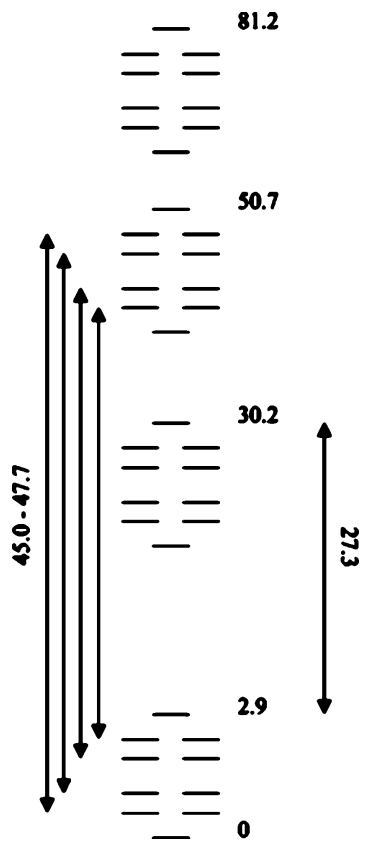

Figure 15. Proposed $\left(\mathrm{D}_{2} \mathrm{O}\right)_{5}$ correlation diagram. One possible scenario to account for the seven observed $\left(\mathrm{D}_{2} \mathrm{O}\right)_{5}$ bands. Energies reported in reciprocal centimeters; drawing not to scale. The three observed bands, $45.0-47.7 \mathrm{~cm}^{-1}$, are assignable within the series of four expected bands linking the lowest and third manifolds.

Furthermore, given that no other $\left(\mathrm{D}_{2} \mathrm{O}\right)_{5}$ bands have been observed near this frequency, it is conceivable that the $81.2 \mathrm{~cm}^{-1}$ band originates in an excited state, perhaps 27.3 or $30.2 \mathrm{~cm}^{-1}$ above the ground state. We know that this is energetically feasible from the observation of water trimer transitions originating $22.7 \mathrm{~cm}^{-1}$ above the ground state. ${ }^{24}$ However, the 81.2 $\mathrm{cm}^{-1}$ band is reported to not have responded to reservoir heating; therefore, it is more likely to arise from the lowest manifold, and in that case, a lack of observed bands in the surrounding frequency range could be attributed to incomplete spectral coverage due to the scarcity of FIR lasers at slightly lower, but not higher, frequencies. We note that the 27.3 and $30.2 \mathrm{~cm}^{-1}$ unperturbed bands could be accounted for by placing one or both of them in a fourth manifold. Graf et al. predicted the $k=$ $5^{1} \leftarrow 0^{0}$ transitions to have near zero intensity regardless of barrier height, ${ }^{31}$ so the $27.3 \mathrm{~cm}^{-1}$ band may arise from $k=0^{1}$ $\leftarrow 5^{0}$ instead of from $k=5^{1} \leftarrow 0^{0}$, which would require the lowest manifold to have a width of $2.9 \mathrm{~cm}^{-1}$. Several Coriolis perturbed bands would then be expected near $30 \mathrm{~cm}^{-1}$. Recall, however, that the 27.3 and the $30.2 \mathrm{~cm}^{-1}$ are both very weak bands and that much stronger trimer transitions, in the form of multiplets, dominate this spectral region. These Coriolis perturbed bands might thereby go unnoticed, especially given sparse laser coverage. A graphical depiction of the proposed water pentamer correlation diagram is given in Figure 15.

There is one major caveat to such a scenario. Many lowfrequency $\mathrm{D}_{2} \mathrm{O}$ pentamer bands, below $10 \mathrm{~cm}^{-1}$, might then be expected. The frequency region between 2 and $4 \mathrm{~cm}^{-1}$ was recently scanned with powerful microwave radiation sources, ${ }^{41}$ and no pentamer bands were observed. Nonetheless, a bandwidth of $2.9 \mathrm{~cm}^{-1}$ would still place many of the expected bands below the covered region. Furthermore, an enormous line density of strong (10 000:1) water dimer transitions dominate this region, such that water pentamer transitions could go unnoticed, especially given the reduced pentamer cluster density (viz., five times lower) in our source as compared to the dimer. ${ }^{42}$ Moreover, the many low-frequency states of a compact lower manifold would be similarly populated, effectively lowering the intensities of the $\mathrm{D}_{2} \mathrm{O}$ pentamer bands linking them.

One other very important piece of information supports our proposed water pentamer correlation diagram: the $\mathrm{H}_{2} \mathrm{O}$ pentamer data. We mentioned that Graf et al. determined that the total pseudorotational, mass-weighted path length, while longer for $\left(\mathrm{D}_{2} \mathrm{O}\right)_{5}$ than for $\left(\mathrm{H}_{2} \mathrm{O}\right)_{5}$, increased by only a factor of 1.30 . We now scale the transition frequencies of the two observed $\left(\mathrm{H}_{2} \mathrm{O}\right)_{5}$ bands using this reduced factor to determine the expected $\left(\mathrm{D}_{2} \mathrm{O}\right)_{5}$ transition frequencies. Scaling of the $89.0 \mathrm{~cm}^{-1}$ band results in an expected $\left(\mathrm{D}_{2} \mathrm{O}\right)_{5}$ transition frequency of $52.7 \mathrm{~cm}^{-1}$. This is very near the experimentally determined $50.7 \mathrm{~cm}^{-1}$ frequency, and indeed, both bands are parallel and unperturbed. Furthermore, the scaled frequency is slightly higher than the observed frequency, just as found for the trimer. Scaling of the non-Coriolis perturbed, parallel $103.8 \mathrm{~cm}^{-1}$ band results in an expected $\left(\mathrm{D}_{2} \mathrm{O}\right)_{5}$ transition frequency of $61.4 \mathrm{~cm}^{-1}$. While no $\left(\mathrm{D}_{2} \mathrm{O}\right)_{5}$ band has yet been observed at this frequency, it would fit quite nicely into the proposed water pentamer correlation diagram, originating in any of the three lowest nondegenerate states and terminating in the fourth $\left(81.2 \mathrm{~cm}^{-1}\right)$ manifold.

Even though a definitive, unambiguous description of the torsional-puckering energy manifold for the water pentamer has not yet emerged, a greatly improved understanding of the water pentamer dynamics has. While clearly similar to the trimer in its facile structural interconversions via torsion or flipping of the free hydrogen atoms, the pentamer differs in that its facile torsional motion is strongly coupled to its less facile oxygen framework puckering motion. The ground-state wave function is probably delocalized over all ten isomeric versions of the global minimum on the rotational time scale, regardless of whether the torsional-puckering pseudorotation itself is excited. This is in agreement with the experimental FIR data, wherein a rigorous oblate symmetric top structure is observed. Nonetheless, a pseudorotational manifold is thought to give rise to all seven observed bands, although it may be formed in part by purely torsional modes. A full treatment of the dynamics will require a ten-dimensional torsional-puckering potential energy surface (PES) and the solution of the complete 10-D coupled anharmonic vibrational Hamiltonian at the very least. The heavy motion of the oxygen atom framework appears to increase the mass-weighted pseudorotational path length of the pentamer well beyond that of the trimer. This results in lower pseudorotational frequencies and a compacting of the torsional-puckering manifolds, consistent with a high density of bands. Observation of additional pentamer bands, in particular, perpendicular bands and bands from the lowest torsional-puckering manifold, remains necessary to definitively specify the correlation diagram of the water pentamer and to obtain a global fit of all observed bands.

Acknowledgment. This work was supported by the Experimental Physical Chemistry Program of the National Science Foundation

Supporting Information Available: Tables VS, VIS, IXS, XS, and XIS report observed transition frequencies and residuals for the five newly fit water pentamer bands; Tables VIIIS and XIIIS report correlation matrices for each of the seven $\left(D_{2} \mathrm{O}\right)_{5}$ bands fit to date. This material is available free of charge via the Internet at http://pubs.acs.org.

\section{References and Notes}

(1) Keutsch, F. N.; Saykally, R. J. Proc. Natl. Acad. Sci. U. S. A. 2001, 98, 10533. 

271,62

2) Liu, K.; Brown, M. G.; Cruzan, J. D.; Saykally, R. J. Science 1996

(3) Ohmine, I. J. Phys. Chem. 1995, 99, 6767.

(4) Wales, D. J.; Hodges, M. P. Chem. Phys. Lett. 1998, 286, 65.

(5) Ma, B. Q.; Sun, H. L.; Gao, S. Chem. Commun. 2004, 2220.

(6) Burnham, C. J.; Xantheas, S. S.; Miller, M. A.; Applegate, B. E.;

Miller, R. E. J. Chem. Phys. 2002, 117, 1109.

(7) Paul, J. B.; Provencal, R. A.; Chapo, C.; Petterson, A.; Saykally, R. J. J. Chem. Phys. 1998, 109, 10201.

(8) Paul, J. B.; Provencal, R. A.; Chapo, C.; Roth, K.; Caseas, R.; Saykally, R. J. J. Phys. Chem. A 1999, 103, 2972.

(9) Xantheas, S. S.; Dunning, T. H., Jr. J. Chem. Phys. 1993, 99, 8774

(10) Hartke, B.; Schutz, M.; Werner, H. J. Chem. Phys. 1998, 239, 561.

(11) Cruzan, J. D.; Viant, M. G.; Brown, M. G.; Lucas, D. D.; Kun, L.; Saykally, R. J. Chem. Phys. Lett. 1998, 292, 667.

(12) Brown, M. G.; Keutsch, F. N.; Saykally, R. J. J. Chem. Phys. 1998 109,9645 .

(13) Liu, K.; Brown, M. G.; Cruzan, J. D.; Saykally, R. J. J. Phys. Chem. A 1997, 101, 9011 .

(14) Busarow, K. L.; Cohen, R. C.; Blake, G. A.; Laughlin, K. B.; Lee,

Y. T.; Saykally, R. J. J. Chem. Phys. 1989, 90, 3937.

(15) Cruzan, J. D.; Braly, L. B.; Liu, K.; Brown, M. G.; Loeser, J. G.; Saykally, R. J. Science 1996, 271, 59.

(16) Liu, K.; Brown, M. G.; Carter, C.; Saykally, R. J.; Gregory, J. K.;

Clary, D. C. Nature (London) 1996, 381, 501

(17) Wales, D. J. Science 1996, 271, 925

(18) Wales, D. J.; Walsh, T. R. J. Chem. Phys. 1996, 105, 6957.

(19) Wales, D. J. Rearrangements and Tunneling Splittings in Small Water Clusters. In Recent Theoretical and Experimental Advances in Hydrogen Bonded Clusters; Xantheas, S., Ed.; Kluwer Academic Publishers: Norwell, 2000; p 201.

(20) Walsh, T. R.; Wales, D. J. J. Chem. Soc., Faraday Trans. 1996 $92,2505$.

(21) Taketsugu, T.; Wales, D. J. Mol. Phys. 2002, 100, 2793.

(22) Wales, D. J.; Taketsugu, T. Private communication.

(23) Keutsch, F. N.; Cruzan, J. D.; Saykally, R. J. Chem. Rev. 2003 , $103,2533$.
(24) Brown, M. G.; Keutsch, F. N.; Braly, L. B.; Saykally, R. J. J. Chem Phys. 1999, 111, 7801.

(25) Liu, K.; Loeser, J. G.; Elrod, M. J.; Host, B. C.; Rzepiela, J. A.; Pugliano, N.; Saykally, R. J. J. Am. Chem. Soc. 1994, 116, 3507.

(26) Schutz, M.; Burgi, T.; Leutwyler, S.; Burgi, H. B. J. Chem. Phys. 1993, 99, 5228 .

(27) van der Avoird, A.; Olthof, E. H. T.; Wormer, P. E. S. J. Chem. Phys. 1996, 105, 8034 .

(28) Olthof, E. H. T.; van der Avoird, A.; Wormer, P. E. S.; Liu, K.; Saykally, R. J. J. Chem. Phys. 1996, 105, 8051.

(29) Viant, M. R.; Brown, M. G.; Cruzan, J. D.; Saykally, R. J.; Geleijns, M.; van der Avoird, A. J. Chem. Phys. 1999, 110, 4369.

(30) Brown, M. G.; Viant, M. R.; McLaughlin, R. P.; Keoshian, C. J.; Michael, E.; Cruzan, J. D.; Saykally, R. J.; van der Avoird, A. J. Chem. Phys. 1999, 111, 7789.

(31) Graf, S.; Mohr, W.; Leutwyler, S. J. Chem. Phys. 1999, 110, 7893

(32) Gregory, J. K.; Clary, D. C. J. Chem. Phys. 1996, 105, 6626.

(33) Cruzan, J. D. Ph.D. Thesis, University of California, Berkeley, 1997.

(34) Keutsch, F. N.; Braly, L. B.; Brown, M. G.; Harker, H. A.; Petersen,

P. B.; Leforestier, C.; Saykally, R. J. J. Chem. Phys. 2003, 119, 8927.

(35) Keutsch, F. N.; Brown, M. G.; Petersen, P. B.; Saykally, R. J.;

Geleijns, M.; van der Avoird, A. J. Chem. Phys. 2001, 114, 3994.

(36) Blake, G. A.; Laughlin, K. B.; Cohen, R. C.; Busarow, K. L.; Gwo,

D.-H.; Schmuttenmaer, C. A.; Steyert, D. W.; Saykally, R. J. Rev. Sci. Instrum. 1991, 62, 1693.

(37) Blake, G. A.; Laughlin, K. B.; Cohen, R. C.; Busarow, K. L.; Gwo,

D.-H.; Schmuttenmaer, C. A.; Steyert, D. W.; Saykally, R. J. Rev. Sci. Instrum. 1991, 62, 1701 .

(38) Liu, K.; Fellers, R. S.; Viant, M. R.; McLaughlin, R. P.; Brown,

M. G.; Saykally, R. J. Rev. Sci. Instrum. 1996, 67, 410.

(39) Herriot, D. R.; Schulte, H. J. Appl. Opt. 1965, 4, 883.

(40) Teller, E. Jahrb. Chem. Physik. 1934, 9, 43.

(41) Keutsch, F. N.; Goldman, N.; Karyakin, E. N.; Harker, H. A.; Sanz,

M. E.; Leforestier, C.; Saykally, R. J. Faraday Discuss. 2001, 118, 79

(42) Paul, J. B.; Collier, C. P.; Saykally, R. J.; Scherer, J. J.; O’Keefe, A. J. Phys. Chem. 1997, 101, 5211. 